\title{
Configurations of infinitely near points
}

\section{A. Campillo}

Departamento de Algebra, Geometria y Topologia, Facultad de Ciencias, Universidad de Valladolid, Prado de la Magdalena s/n, 47005 Valladolid, Spain.

E-mail address: campillo@agt.uva.es

\section{G. Gonzalez-Sprinberg}

Institut Fourier, Université Grenoble I, BP 74, 38402 Saint-Martin-d'Hères, France.

E-mail address: gonsprin@ujf-grenoble.fr

\section{F. Monserrat ${ }^{1}$}

Instituto Universitario de Matemática Pura y Aplicada, Universidad Politécnica de Valencia, Camino de Vera s/n, 46022 Valencia, Spain.

E-mail address: framonde@mat.upv.es

\begin{abstract}
We present a survey of some aspects and new results on configurations, i.e. disjoint unions of constellations of infinitely near points, local and global theory, with some applications and results on generalized Enriques diagrams, singular foliations, and linear systems defined by clusters.

1991 Mathematics Subject Classification: 14C20, 14M25, $13 \mathrm{~B} 22$.

Keywords and phrases: infinitely near points, proximity relations, Enriques diagrams, clusters, characteristic cones, complete ideals, toric varieties.
\end{abstract}

\section{Introduction}

The subject in this paper can be understood to be originated with the study of singularities of plane curves, their desingularization by iterated point blowing-ups, and the problems of existence of curves having either assigned singularities or passing through a given set of points or infinitely near points with prescribed multiplicities.

\footnotetext{
${ }^{1}$ Supported by MEC MTM2007-64704 and Bancaixa P1-1A2005-08.
} 
Early, in the past century, Enriques gave an answer for the existence of such curves, without conditions on the degree, in terms of precise inequalities involving the prescribed multiplicities. Some essential data for it are the proximity relations among the given infinitely near points, i.e. the incidence between points and the transforms of exceptional divisors obtained by blowing-up precedent points. This data is encoded in the so called Enriques diagrams.

Later, the local study of (complete) linear systems of curves leads Zariski, in the thirties, to define complete ideals on regular local rings, investigate their structure and establish their theory in the smooth two dimensional case. Lipman has continued the development of the theory of complete ideals for singular two dimensional cases and in higher dimensions; in particular, in the eighties, by establishing it for finitely supported complete ideals, i.e. for ideals supported at the closed point and such that there exist finite sequences of point blowing-ups which make the ideals locally principal. In parallel, also in the later eighties, Casas develops in modern geometrical terms the two dimensional theory, and applies it to solve the problem of determining multiplicities of passage through infinitely near points for polar curves of plane curve singularities.

Factorization theorems are obtained, in general, for these ideals in terms of simple (or special *-simple) ideals corresponding to finite chains of infinitely near points. An algebraic-geometric point of view, in terms of geometry of infinitely near points, was given by the first two authors and Lejeune-Jalabert in the nineties. This includes the treatment of finitely supported toric ideals, and a rather explicit theory for them.

Applications of finitely supported complete ideals have been developed, both in the local and global cases, by means of constellations and configurations of infinitely near points respectively. Such applications include those of different subjects as, among others, the study of singularities of adjoints or polar curves [13], [14], [21], [52], [50], the Poincaré problem on the degree of projective integral curves of first order algebraic differential equations [5],[6],[7],[23],[47],[29], the Harbourne-Hirschowitz conjecture on special linear systems of projective plane curves (and related topics) [61], [62], [63], [56], [54], or the monodromy and related conjectures for non degenerated hypersurfaces with respect to clusters, [45], [46].

This paper gives an introduction to this subject as well as a survey on the main results and several of its applications. Some new results are also included.

The paper is organized as follows. 
In Section 2 we give a survey on the local aspects of the theory. After recalling the basic definitions of the geometric theory extending to higher dimensions the classical case presented in [22], we relate this geometric framework with the algebraic theory introduced in [70, 71]. It follows the Lipman's unique factorization result (allowing negative exponents) for finitely supported complete ideals [49] on a germ of smooth variety, which extends to higher dimensions the unique factorization of any complete ideal of a two dimensional local ring into simple ideals. Each simple ideal in the factorization is associated to one infinitely near point to the origin, and it reflects geometrical properties of the chain of points which need to be blown up in order to create it. A union of such chains with the same origin will be called constellation along the paper. Constellations with integral weights at their points are called clusters. Complete ideals supported on a constellation correspond to concrete clusters called idealistic. Such clusters with the natural semigroup structure are called the Galaxy of the constellation. Galaxies become closed under taking adjoints, and the part consisting of idealistic clusters corresponding to adjoint ideals is determined from the Galaxy itself.

Assuming that the characteristic of the ground field is zero, the morphism $\sigma_{\mathcal{C}}: X_{\mathcal{C}} \rightarrow X$ obtained by composition of the blowing-up of the points of a constellation $\mathcal{C}$ on the smooth germ $X$ is shown to be an embedded resolution of complete intersections defined by general elements in a finitely supported complete ideal with that constellation as support. The variety $X_{\mathcal{C}}$ will be called the sky of $\mathcal{C}$. On the other hand, in Section 2.4 it is shown how the use of characteristic cones provides a natural framework to study factorizations properties for ideals.

Finally, we consider the case of toric varieties and monomial ideals, giving explicit description and results on toric constellations and proximity in combinatorial terms, which are not available in general non toric cases. Linear proximity, a finer concept than the one of proximity, is also characterized and used for describing the Galaxy, the characteristic cone and factorization properties. For the ideals in the subgalaxy generated by the simple factors in Lipman factorization the whole features of Zariski's theory hold as in the smooth two dimensional case. Generalized Enriques diagrams, i.e. those which also contain the linear proximity information, are also characterized.

Section 3 is devoted, on the one hand, to extend the language and theory of constellations of infinitely near points and clusters to the global situation. Here, we consider configurations, i.e. finite union of constellations with origin at different points of a smooth variety. The notions of sky, clusters and Galaxy have an obvious sense also for configurations. On the other hand, we focus our attention on the study of the cone of curves $N E(Z)$ of a 
projective regular surface $Z$, mainly bearing in mind the case in which $Z$ is rational. We give a description of some generalities and known properties of $N E(Z)$, showing a variety of different shapes that it may have, and, in particular, we focus on the case of finite generation (polyhedrality). Rational surfaces with polyhedral cone of curves are interesting issues that have several applications, as we shall see in the last section.

The so called P-sufficient configurations, introduced in [27] and [28], are configurations over a relatively minimal rational surface $X$ satisfying a numerical condition which depends only on their P-Enriques diagram. The interest of such a configuration $\mathcal{C}$ is given by the fact that its sky $X_{\mathcal{C}}$ has a polyhedral cone of curves. We consider also configurations $\mathcal{C}$ of base points of 1 -dimensional linear systems (pencils) $\varrho$ on a projective regular surface. We give a description of the face of $N E\left(X_{\mathcal{C}}\right)$ generated by the classes of the integral components of the curves in $\varrho$, using it to give (in characteristic zero) a characterization of the irreducible pencils (that is, those with integral general curves) in terms of their clusters of base points. When $\varrho$ is a pencil at infinity a great deal of information is known on the cone of curves and the characteristic cone of $X_{\mathcal{C}}[10,11]$ and we summarize it.

In Section 4 we show some of the above mentioned applications of the theory of clusters of infinitely near points in the global case. In Section 4.1 we describe some results on the theory of foliations based on aspects in preceding sections. All these results are related to the classical Poincaré problem on establishing bounds for the degree of projective curves which are invariant by an algebraic plane foliation. In Section 4.2 we show that the language of infinitely near points and idealistic clusters can also be applied to give new results on a conjecture (the Harbourne-Hirschowitz Conjecture) which deals with the dimension of the linear systems of the projective plane defined by clusters whose associated configuration is a set of general points of the plane. In both cases, there is a very extensive literature giving either partial proofs for problem or for the conjecture or dealing with related subjects. We recall some results in cases for which the Poincaré problem or the Harbourne-Hirschowitz Conjecture have a satisfactory answer. Those results are established in terms of clusters and their proofs involve the knowledge of the cone of curves of the surfaces associated with certain pencils at infinity and certain properties of the P-sufficient configurations.

\section{Local theory}

2.1. Constellations, proximity and Enriques diagrams. Let $X$ be a regular variety of dimension $d$ at least two, over an algebraically closed field $\mathbb{K}$. In the sequel we consider varieties obtained from $X$ by a finite sequence 
of closed point blowing-ups. A point $P$ is infinitely near $Q \in X$ if $Q$ is the image of $P$ under the composition of blowing-ups; denote this relation by $P \geq Q$. A geometric description of the blowing-up of a point may be given as an avatar of the graph construction related to the definition of projective space. Let $\varphi:\left(\mathbb{K}^{d} \backslash\{O\}\right) \rightarrow \mathbb{P}^{d-1}$ with $\varphi(x)$ the line joining $O$ to $x$. Consider the closure $\bar{\Gamma}_{\varphi}$ of the graph of $\varphi$ in $\mathbb{K}^{d} \times \mathbb{P}^{d-1}$. The blowing-up of $\mathbb{K}^{d}$ with center $O$ is the proper birational morphism given by the projection on the first factor $\sigma: B l_{0} \mathbb{K}^{d}:=\bar{\Gamma}_{\varphi} \rightarrow \mathbb{K}^{d}$. The exceptional fiber over $O$ is a rational divisor $B_{0} \simeq \mathbb{P}^{d-1}$.

Definition 2.1. A constellation of infinitely near points (in short, a constellation) is a set $\mathcal{C}=\left\{Q_{0}, \ldots, Q_{n}\right\}$, with $Q_{i} \geq Q_{0} \in X_{0}=X$, such that $Q_{i} \in B l_{Q_{i-1}} X_{i-1}=: X_{i} \stackrel{\sigma_{i-1}}{\longrightarrow} X_{i-1}$, for $1 \leq i \leq n$; where $B l_{Q_{i-1}} X_{i-1}$ denotes the blowing-up of $X_{i-1}$ with center $Q_{i-1}$.

The point $Q_{0}$ is called the origin of the constellation $\mathcal{C}$. We call also the dimension of $X$ the dimension of $\mathcal{C}$. Let $\sigma_{\mathcal{C}}=\sigma_{0} \circ \cdots \circ \sigma_{n}: X_{\mathcal{C}} \rightarrow X_{0}$ denote the composition of the blowing-ups of all the points of $\mathcal{C}$, where $X_{\mathcal{C}}=X_{n+1}$. Two constellations $\mathcal{C}$ and $\mathcal{C}^{\prime}$ over $X$ are identified if there is an automorphism $\pi$ of $\mathrm{X}$ and an isomorphism $\pi^{\prime}: X_{\mathcal{C}} \rightarrow X_{\mathcal{C}^{\prime}}$ such that $\sigma_{\mathcal{C}^{\prime}} \circ \pi^{\prime}=\pi \circ \sigma_{\mathcal{C}}$. The relation $Q_{j} \geq Q_{i}$ is a partial ordering on the set of points of $\mathcal{C}$. If this ordering is total, i.e. $Q_{n} \geq \cdots \geq Q_{0}$, we say that $\mathcal{C}$ is a chain constellation. For example, for any constellation $\mathcal{C}$ and any $Q \in \mathcal{C}$, the set $\mathcal{C}^{Q}:=\{P \in \mathcal{C} \mid Q \geq P\}$ of points preceding $Q$ is a chain constellation. The number of points in $\mathcal{C}^{Q}$, different from $Q$, is called the level of $Q$. The root of $C$ is the only point of level 0 . For each point $Q \in \mathcal{C}$ let $Q^{+}$be the set of points of $\mathcal{C}$ consecutive to $Q$, i.e. the points following $Q$ for the ordering $\geq$ such that there is no strict intermediate point; write $\left|Q^{+}\right|$for the cardinal of this set. If $Q^{+}$has only one point, it denotes this point. For each point $Q=Q_{i}$, let $B_{Q}$ (or $B_{i}$ ) be the exceptional divisor $\sigma_{i}^{-1}(Q)$ on $X_{i+1}$, and $E_{Q}$ (or $E_{i}$ ) its successive strict (or proper) transforms on any $X_{j}$ (which will be specified if necessary) with $Q_{j} \geq Q_{i}$, in particular in $X_{\mathcal{C}}$. The total transforms are denoted by $E_{Q}^{*}$ or $E_{i}^{*}$. The sets of divisors $\left\{E_{Q} \mid Q \in \mathcal{C}\right\}$ and $\left\{E_{Q}^{*} \mid Q \in \mathcal{C}\right\}$, considered in $X_{\mathcal{C}}$, are two basis of the lattice $N^{1}=\bigoplus_{Q \in \mathcal{C}} \mathbb{Z} E_{Q} \cong \mathbb{Z}^{n+1}$ of divisorial cycles with exceptional support in $X_{\mathcal{C}}$.

Definition 2.2. A point $Q_{j} \geq Q_{i}$ is proximate to $Q_{i}$ if $Q_{j} \in E_{i}$ in $X_{j}$; notation : $Q_{j} \rightarrow Q_{i}$ (or $j \rightarrow i$ ). The proximity index of a point $Q_{j}$ is defined as the number $\operatorname{ind}\left(Q_{j}\right)$ of points in $\mathcal{C}$ approximated by $Q_{j}$, i.e. $\operatorname{ind}\left(Q_{j}\right):=\#\left\{Q_{i} \in \mathcal{C} \mid Q_{j} \rightarrow Q_{i}\right\}$. 
If $R \in Q^{+}$then $R \rightarrow Q$, these are the so called trivial proximities. If $R$ belongs to the intersection of several exceptional divisors produced by blowing-up precedent points then $R$ is proximate to all these points. Since the irreducible exceptional divisors we consider have normal crossing, in dimension $d$ a point may be proximate to at most $d$ points. If the dimension of $C$ is at least three, then $R \rightarrow Q$ if and only $R \geq Q$ and $E_{R} \cap E_{Q} \neq \emptyset$ in $X_{\mathcal{C}}$. Note that if $R \rightarrow Q$ then $R \geq Q$, but the converse does not hold in general. The proximity relation $(\rightarrow)$ is a binary relation on the set of points of a constellation, but not an ordering in general.

Remark 2.1. For each point $Q_{i}$, the only irreducible exceptional divisors, besides $E_{i}$, appearing in the total transform $E_{i}^{*}$, in $X_{\mathcal{C}}$, are exactly those produced by blowing-up the points proximate to $Q_{i}$. Therefore $E_{i}=E_{i}^{*}-$ $\sum_{j \rightarrow i} E_{j}^{*}$. The so called proximity matrix $\left(\left(p_{j i}\right)\right)$, with $p_{i i}=1, p_{j i}=-1$ if $j \rightarrow i$ and 0 otherwise, is the basis change matrix from the $E_{i}$ 's to the $E_{j}^{*}$ 's

Definition 2.3. The (proximity) Enriques diagram or P-Enriques diagram of a constellation $\mathcal{C}$ is the rooted tree $\Gamma_{\mathcal{C}}$ equipped with a binary relation $(\sim)$, whose vertices are in one to one correspondence with the points of $\mathcal{C}$, the edges with the couples of points $(R, Q)$ such that $R \in Q^{+}$, the root with the origin of $\mathcal{C}$, and the relation $(\sim)$ with the proximity relation $(\rightarrow)$.

The Enriques diagram codes the chronology and incidence data of the points in a constellation. Any (finite) rooted tree (without the supplementary data of a binary relation) may be the support graph of an Enriques diagram.

Remark 2.2. There is another graph in dimension two that may be associated to the (normal crossing) family of irreducible exceptional divisors obtained by blowing-up the points of a constellation. This is the so called dual graph, whose vertices are in bijection with the divisors and each edge is associated to the intersection point of two divisors. The graph supporting the Enriques diagram of a constellation and the dual graph of the exceptional divisors may be quite different. For instance the first one may be a chain but not the other, or viceversa.

A natural question is how to characterize the Enriques diagrams, i.e. which rooted trees equipped with a binary relation on the set of vertices are induced by some constellation. Given a rooted tree $\Gamma$, denote by $(\succeq)$ the natural partial ordering on the set $\mathcal{V}(\Gamma)$ of its vertices : $p \succeq q$ if $q$ belongs to the chain from $p$ to the root; similarly, if $(\sim)$ is a binary relation on $\mathcal{V}(\Gamma)$, let $\operatorname{ind}(q)=\#\{p \in \mathcal{V}(\Gamma) \mid q \leadsto p\}$. For each vertex $q$, let $q^{+}$be the set of consecutive vertices to $q$ with respect to the ordering $(\succeq)$.

Theorem 2.1. Let $\Gamma$ be a finite rooted tree equipped with a binary relation $(\leadsto)$ on the set of its vertices. Then $\Gamma$ is the Enriques diagram of a 
constellation of infinitely near points $\mathcal{C}$ and $(\sim)$ is induced by the proximity relation on $\mathcal{C}$ if and only if, for any vertices $p, q, r$ of $\Gamma$, the following conditions are satisfied:

(a) $q \leadsto p \quad \Longrightarrow q \succeq p, q \neq p$

(b) $q \in p^{+} \Longrightarrow q \leadsto p$

(c) $r \succeq p \succeq q$ and $r \leadsto q \Longrightarrow p \leadsto q$

If these conditions hold, then the minimum dimension $d_{\mathcal{P}}$ of a constellation whose Enriques diagram is the given one is at most

$\max \left(2, \max _{q \in \mathcal{V}(\Gamma)}(\operatorname{ind}(q))+1\right)$.

Proof. The necessity of the conditions follows easily. For the sufficiency, proceed by induction on the number $|\mathcal{V}(\Gamma)|$ of vertices. If $|\mathcal{V}(\Gamma)|>1$, let $r$ be a maximal vertex of $\Gamma$, and assume that a constellation $\mathcal{C}^{\prime}$ of dimension $d$ works for $\Gamma^{\prime}=\Gamma \backslash\{r\}$. Let $r \in q^{+}$, and $Q$ be the point of $\mathcal{C}^{\prime}$ corresponding to $q$. The set $Y:=\left\{P \in \mathcal{C}^{\prime} \mid r \leadsto p\right\}$ is contained in $\mathcal{C}^{\prime Q}$ by (a) and $Q \in Y$ by (b). By (c) one has $Q \rightarrow P$ for each $P \in Y \backslash\{Q\}$, so that $Q \in F:=\bigcap_{P \in Y, P \neq Q} E_{P}$. It follows that $F \neq \emptyset$ and $\operatorname{dim}(F)=d+1-|Y|$, by the normal crossing of the divisors $E_{P}$, and on the other hand $\operatorname{ind}(Q) \geq$ $|Y \backslash\{Q\}|=|Y|-1$. Now, we need a point $R$ (in $X_{\mathcal{C}^{\prime}}$ ) corresponding to $r$, having the corresponding proximities, i.e. a point $R \in B_{Q} \bigcap F$ but not in $\left(Q^{+} \bigcup_{P \in \mathcal{C}^{Q} \backslash Y} E_{P}\right)$. Such a point exists if $d \geq \max _{p \in \mathcal{V}(\Gamma)} i n d(p)+1$ (and at least 2), which is not less than $\max _{p \in \mathcal{V}\left(\Gamma^{\prime}\right)} i n d(p)+1$ so the inductive hypothesis applies. This number is attained.

Remark 2.3. The minimum dimension $d_{\mathcal{P}}$ of constellations inducing a given P-Enriques diagram may be one less than in the general case if there are no two maximal vertices $r$, with maximum indices, say $r_{1}$ and $r_{2}$, both in $q^{+}$, such that $i n d\left(r_{i}\right)=\operatorname{ind}(q)+1$. Precisely, the minimum dimension is $d_{\mathcal{P}}=\max \left(2, \max _{q \in \mathcal{V}(\Gamma)}(\operatorname{ind}(q)+t(q))\right)$, where $t(q)=0($ resp. $t(q)=1)$ if $s(q):=\#\left\{r \in q^{+} \mid \operatorname{ind}(r)>\operatorname{ind}(q)\right\} \leq 1$ (resp. if $s(q) \geq 2$ ).

\subsection{Finitely supported ideals and idealistic clusters.}

Definition 2.4. A cluster is a pair $\mathcal{K}=(\mathcal{C}, \underline{m})$ where $\mathcal{C}=\left\{Q_{0}, \ldots, Q_{n}\right\}$ is a constellation and $\underline{m}=\left(m_{0}, \ldots, m_{n}\right)$ is a sequence of integers. The integer $m_{i}$ is called the weight (or virtual multiplicity) of $Q_{i}$ in the cluster.

Given a cluster $\mathcal{K}$ as above, we can associate to it the following divisor in $X_{\mathcal{C}}$ with exceptional support: $D(\mathcal{K}):=\sum_{i=0}^{n} m_{i} E_{i}^{*}$. Hence, given a constellation $\mathcal{C}$, the choice of a weight sequence $\underline{m}$ is equivalent to the choice of a divisor in the semigroup $\sum_{i=0}^{n} \mathbb{Z}_{\geq 0} E_{i}^{*}$. 
Definition 2.5. Given a closed point $Q_{0} \in X$, an ideal $I$ in $R_{Q_{0}}:=\mathcal{O}_{X, Q_{0}}$ is finitely supported if $I$ is primary for the maximal ideal $M_{Q_{0}}$ of $R_{Q_{0}}$ and there exists a constellation $\mathcal{C}$ of infinitely near points of $X$ such that $I \mathcal{O}_{X_{\mathcal{C}}}$ is an invertible sheaf. An infinitely near point $P$ of $Q_{0}$ is a base point of $I$ if $P$ belongs to the constellation with the minimal number of points with the above property. We shall denote by $\mathcal{C}_{I}$ the constellation of base points of $I$.

Given a finitely supported ideal $I$ in $R_{Q_{0}}=\mathcal{O}_{X, Q_{0}}$, with associated constellation of base points $\mathcal{C}_{I}=\left\{Q_{0}, \ldots, Q_{n}\right\}$, we can associate to it a cluster $\mathcal{K}=\mathcal{K}_{I}=\left(\mathcal{C}_{I}, \underline{m}\right)$, called cluster of base points of $I$, as we shall describe now. For any point $Q_{i}, 0 \leq i \leq n$, consider the chain constellation of preceding points $\mathcal{C}^{Q_{i}}=\left\{P_{0}=Q_{0}, P_{1}, \ldots, P_{r}=Q_{i}\right\}$; the weak transforms $I_{P_{j}}$ of $I$ at the points $P_{j}$ are defined by induction on $r$ by setting $I_{Q_{0}}=I$ and, for $i>0, I_{P_{i}}$ is the ideal in the local ring $\left(R_{P_{i}}, M_{P_{i}}\right)$ given by

$$
(x)^{-\operatorname{ord}_{P_{i-1}}\left(I_{P_{i-1}}\right)} I_{P_{i-1}} R_{P_{i}}
$$

where, $\operatorname{ord}_{P_{i-1}}\left(I_{P_{i-1}}\right):=\max \left\{n \mid I_{P_{i-1}} \subseteq M_{P_{i-1}}^{n}\right\}$ and $x$ is a generator of the principal ideal $M_{P_{i-1}} R_{P_{i}}$. For any $i, 0 \leq i \leq n$, the weight $m_{i}$ is defined to be $\operatorname{ord}_{Q_{i}}\left(I_{Q_{i}}\right)$. Notice that the ideal $I_{Q_{i}}$ is finitely supported and $m_{i}>0$. Moreover, it follows by induction the following equality between ideal sheaves on $X_{\mathcal{C}_{I}}$ :

$$
I \mathcal{O}_{X_{\mathcal{C}_{I}}}=\mathcal{O}_{X_{\mathcal{C}_{I}}}\left(-D\left(\mathcal{K}_{I}\right)\right) \text {. }
$$

Remark 2.4. The completion (or integral closure) $\bar{I}$ of a finitely supported ideal $I$ is again finitely supported and $\mathcal{K}_{\bar{I}}=\mathcal{K}_{I}$ (see [49, Prop. 1.10]).

For a fixed constellation $\mathcal{C}$ rooted at $Q_{0} \in X$, we shall denote by $\mathcal{J}_{\mathcal{C}}$ the set of of finitely supported complete ideals $I$ of $\mathcal{O}_{X, Q_{0}}$ such that $\mathcal{C}_{I} \subseteq \mathcal{C}$. This set $\mathcal{J}_{\mathcal{C}}$ can be endowed with an operation, called $*$-product: given $I_{1}, I_{2} \in \mathcal{J}_{\mathcal{C}}, I_{1} * I_{2}$ is defined to be the integral closure of the product ideal $I_{1} I_{2}$. Notice that $\left(\mathcal{J}_{\mathcal{C}}, *\right)$ has structure of commutative semigroup.

Definition 2.6. We shall say that a cluster $\mathcal{K}=(\mathcal{C}, \underline{m})$ is idealistic if there exists a finitely supported ideal $I$ in $R_{Q_{0}}$ such that $I \mathcal{O}_{X_{\mathcal{C}}}=\mathcal{O}_{X_{\mathcal{C}}}(-D(\mathcal{K})$ ). Notice that this implies that $I \in \mathcal{J}_{\mathcal{C}}$ and that $m_{i}$ is the weight of $Q_{i}$ in $\mathcal{K}_{I}$ if $Q_{i} \in \mathcal{C}_{I}$ and $m_{i}=0$ otherwise. The galaxy of $\mathcal{C}$ will be the set $\mathcal{G}_{\mathcal{C}}$ of idealistic clusters on $\mathcal{C}$.

From [48, Sect. 18] it follows a characterization of the idealistic clusters:

Proposition 2.1. A cluster $\mathcal{K}=(\mathcal{C}, \underline{m})$ is idealistic if and only if $\underline{m} \neq 0$ and $-D(\mathcal{K})$ is $\sigma_{\mathcal{C}}$-generated, i.e. $\mathcal{O}_{X_{\mathcal{C}}}(-D(\mathcal{K}))$ is generated by its global sections on a neighbourhood of the exceptional fiber of $\sigma_{\mathcal{C}}: X_{\mathcal{C}} \rightarrow X$. 
As a consequence of this proposition, one has that the galaxy $\mathcal{G}_{\mathcal{C}}$ of a constellation $\mathcal{C}$ has a natural structure of commutative semigroup with the following operation: if $\mathcal{K}_{i}=\left(\mathcal{C}, \underline{m}_{i}\right) \in \mathcal{G}_{\mathcal{C}}, i=1,2, \mathcal{K}_{1}+\mathcal{K}_{2}:=\left(\mathcal{C}, \underline{m}_{1}+\underline{m}_{2}\right)$. Moreover, if $I_{1}, I_{2} \in \mathcal{J}_{\mathcal{C}}$, it is satisfied that $\mathcal{K}_{I_{1} * I_{2}}=\mathcal{K}_{I_{1}}+\mathcal{K}_{I_{2}}$. Also, Proposition 1.10 of [49] shows that, given a constellation $\mathcal{C}$ and an idealistic cluster $\mathcal{K} \in \mathcal{G}_{\mathcal{C}}$, there exists a unique finitely supported complete ideal $I_{\mathcal{K}} \in \mathcal{J}_{\mathcal{C}}$ such that $I_{\mathcal{K}} \mathcal{O}_{X_{\mathcal{C}}}=\mathcal{O}_{X_{\mathcal{C}}}(-D(\mathcal{K}))$; actually, it is the stalk at the root of $\mathcal{C}$ of the sheaf $\sigma_{\mathcal{C} *} \mathcal{O}_{X_{\mathcal{C}}}(-D(\mathcal{K}))$. If we set $\mathbb{E}_{\mathcal{C}}^{\sharp}$ the semigroup of effective divisors $D$ on $X_{\mathcal{C}}$ with exceptional support such that $D \neq 0$ and $\mathcal{O}_{X_{C}}(-D)$ is $\sigma_{\mathcal{C}}$-generated, above considerations are summarized in the following result:

Proposition 2.2. Given a constellation $\mathcal{C}$, the assignments $\mathcal{K} \mapsto D(\mathcal{K})$ and $\mathcal{K} \mapsto I_{\mathcal{K}}$ give isomorphisms of commutative semigroups $\left(\mathcal{G}_{\mathcal{C}},+\right) \rightarrow\left(\mathbb{E}_{\mathcal{C}}^{\sharp},+\right)$ and $\left(\mathcal{G}_{\mathcal{C}},+\right) \rightarrow\left(\mathcal{J}_{\mathcal{C}}, *\right)$ respectively. The inverse maps are defined by the assignments $D \mapsto \mathcal{K}_{J}$ (where $J$ denotes the stalk of $\sigma_{\mathcal{C} *} \mathcal{O}_{X_{\mathcal{C}}}(-D)$ at the origin of $\mathcal{C}$ ) and $I \mapsto \mathcal{K}_{I}$, respectively.

Remark 2.5. Note that, in the above statement, for each ideal $I \in \mathcal{J}_{\mathcal{C}}$ we are identifying the cluster $\mathcal{K}_{I}=\left(\mathcal{C}_{I}, \underline{m}\right)$ with $\left(\mathcal{C}, \underline{m}^{\prime}\right)$, where $m_{i}^{\prime}=m_{i}$ if $Q_{i} \in \mathcal{C}_{I}$ and $m_{i}^{\prime}=0$ otherwise.

Let $\operatorname{Nef}\left(X_{\mathcal{C}} / X\right)$ be the semigroup of non zero $\sigma_{\mathcal{C}}$-nef divisors on $X_{\mathcal{C}}$ (also called either numerically effective or semiample divisors), that is, those exceptional divisors $D \neq 0$ such that $D \cdot C \geq 0$ for any exceptional curve (i.e. effective exceptional irreducible 1-cycle) $C$ on $X_{\mathcal{C}}$.

Proposition 2.3. [9, Prop. 1.22] If $\mathcal{C}$ is a constellation over $X$ then $\mathbb{E}_{\mathcal{C}}^{\sharp} \subseteq$ $-\operatorname{Nef}\left(X_{\mathcal{C}} / X\right)$.

If the dimension $d$ of $X$ equals 2 , then the effective exceptional irreducible 1-cycles of $X_{\mathcal{C}}$ are the strict transforms of the exceptional divisors. Then, a divisor $-D=-\sum_{i=1}^{n} m_{i} E_{i}^{*}$ is $\sigma_{\mathcal{C}}$-nef if and only if $-D \cdot E_{i}=m_{i}-$ $\sum_{j \rightarrow i} m_{j} \geq 0$ for $0 \leq i \leq n$. This inequalities are classically known as proximity inequalities (see [22], Chap. II, book 4). But, in this case, it is also known that if $-D$ is $\sigma_{\mathcal{C}}$-nef then it is $\sigma_{\mathcal{C}}$-generated $[22,48,13$, 51]. Therefore, in dimension 2 , the inclusion given in the statement of Proposition 2.3 is an equality. Hence, we have a satisfactory description of the idealistic clusters: a cluster is idealistic if and only if its weights satisfy the proximity inequalities. If $d>2$ the inclusion given in Proposition 2.3 need not be an equality. This fact is shown in the following example, taken from [9]:

Example 2.1. Let $X$ be a 3 -dimensional non singular variety and let $\mathcal{C}=$ $\left\{Q_{0}, \ldots, Q_{9}\right\}$ a constellation consisting of a closed point $Q_{0} \in X$ and nine 
points $Q_{1}, \ldots Q_{9}$ in general position on a non singular cubic curve $C_{0}$ in the exceptional divisor $B_{0}$ (i.e. such that $C_{0}$ is the unique cubic curve in $B_{0}$ passing through the nine points). Consider the divisor on $X_{\mathcal{C}}$ given by $D=3 E_{0}^{*}+\sum_{i=1}^{9} E_{i}^{*} . \quad-D$ is $\sigma_{\mathcal{C}}$-nef because, if $C$ is any curve in $B_{0}$, the inequality $3 \operatorname{deg}(C)-\sum_{i=1}^{9} e_{Q_{i}}(C) \geq 0\left(e_{Q_{i}}(C)\right.$ denoting the multiplicity of $C$ at $Q_{i}$ ) is obvious if $C=C_{0}$ and it follows from Bézout's theorem otherwise. However, $-D$ is not $\sigma_{\mathcal{C}}$-generated because, if otherwise, $C_{0}$ should be a fixed curve of the finitely supported ideal $I$ such that $I \mathcal{O}_{X_{\mathcal{C}}}=$ $\mathcal{O}_{X_{\mathcal{C}}}(-D)$.

However, the semigroup $\mathbb{E}_{\mathcal{C}}^{\sharp}$ has the property to be closed under adjoints. In fact, if $K_{X_{\mathcal{C}} / X}$ is the relative canonical divisor of the morphism $\sigma_{\mathcal{C}}$ and $\mathcal{K}=(\mathcal{C}, \underline{m})$ is an idealistic cluster, then the adjoint ideal $J_{\mathcal{K}}:=\sigma_{\mathcal{C} *} \mathcal{O}_{X_{\mathcal{C}}}\left(-D(\mathcal{K})+K_{X_{\mathcal{C}} / X}\right)$ of the ideal $I_{\mathcal{K}}$ is again a finitely supported on $\mathcal{C}$ complete ideal, namely the one associated to the cluster with weights $\max \left(0, m_{i}-d+1\right)$ for any $i$. This statement is due to Lipman. Hence, one deduces the following result:

Proposition 2.4. [52, Th. 3.3] For a given constellation $\mathcal{C}$ one has that if $\sum_{i=0}^{n} m_{i} E_{i}^{*} \in \mathbb{E}_{\mathcal{C}}^{\sharp}$ then $\sum_{i=0}^{n} \max \left(0, m_{i}-d+1\right) E_{i}^{*} \in \mathbb{E}_{\mathcal{C}}^{\sharp}$.

Moreover, a given divisor $\sum_{i=0}^{n} m_{i}^{\prime} E_{i}^{*}$ is the associated divisor to the adjoint of some finitely supported ideal if and only if $\sum_{i=0}^{n}\left(m_{i}^{\prime}+d-1\right) E_{i}^{*} \in$ $\mathbb{E}_{\mathcal{C}}^{\sharp}$. This follows from the definition and above result. For $d=2$ this fact was proved in [41, Th. 1].

Definition 2.7. A finitely supported complete ideal $I$ of a local ring $\mathcal{O}_{X, Q_{0}}$ is said to be $*$-simple if it cannot be factorized as $*$-product of two proper ideals of $\mathcal{O}_{X, Q_{0}}$ or, equivalently, $I$ is not the $*$-product of two proper ideals belonging to $\mathcal{J}_{\mathcal{C}}$, whenever $I \in \mathcal{J}_{\mathcal{C}}$ for a constellation $\mathcal{C}$.

Remark 2.6. If $d=2$ the product of complete ideals is a complete ideal and, hence, the operation $*$ coincides with the usual product of ideals; in this case, the $*$-simple complete ideals are called simple complete ideals.

In [49] Lipman associates, to each point $Q_{j}$ of a constellation $\mathcal{C}=$ $\left\{Q_{0}, \ldots, Q_{n}\right\}$, the unique finitely supported complete $*$-simple ideal $\mathcal{P}_{Q_{j}}$ of $R_{Q_{0}}$ whose cluster of base points $\mathcal{K}_{\mathcal{P}_{Q_{j}}}=\left(\mathcal{C}_{\mathcal{P}_{Q_{j}}}, \underline{m}\right)$ satisfies the conditions: $\mathcal{C}_{\mathcal{P}_{Q_{j}}}=\mathcal{C}^{Q_{j}}$, the weight of $Q_{j}$ equals 1 and the weight sequence $\underline{m}$ is minimal for the reverse lexicographical ordering in $\left(\mathbb{Z}_{\geq 0}\right)^{\ell+1}$, where $\ell$ is the level of $Q_{i}$. For simplicity of notation, we shall denote by $D\left(Q_{j}\right)$ the 
divisor on $X_{\mathcal{C}}$ given by $D\left(\mathcal{K}_{\mathcal{P}_{Q_{j}}}\right)$, that is:

$$
D\left(Q_{j}\right):=\sum_{Q_{i} \leq Q_{j}} m_{i j} E_{i}^{*}
$$

where $m_{i j}$ is the virtual multiplicity of $Q_{i}$ in the cluster $\mathcal{K}_{\mathcal{P}_{Q_{j}}}$. Since $m_{j j}=$ 1 for all $j$, one has that the set $\left(D\left(Q_{0}\right), \ldots, D\left(Q_{n}\right)\right)$ is a basis of $N^{1}$ and the basis change matrix from $\left(D\left(Q_{i}\right)\right)$ to $\left(E_{i}^{*}\right)$ is the matrix $\mathbf{M}_{\mathcal{C}}:=\left(\left(m_{i j}\right)\right)$. As a consequence of this fact and Proposition 2.2 we get the Lipman's unique factorization theorem (see [49]):

Theorem 2.2. Given a constellation $\mathcal{C}=\left\{Q_{0}, \ldots, Q_{n}\right\}$, for each $I \in \mathcal{J}_{\mathcal{C}}$ we can write formally, in a unique form, the ideal $I$ as $*$-product of the *-simple ideals $\mathcal{P}_{Q_{i}}$ associated with the points in $\mathcal{C}$ :

$$
I=\prod_{0 \leq i \leq n}^{*} \mathcal{P}_{Q_{i}}^{r_{i}}
$$

with $r_{i} \in \mathbb{Z}$ for all $i=0, \ldots, n$. Moreover, the vector $\underline{r}=\left(r_{1}, \ldots, r_{n}\right)$ can be computed as $\underline{r}^{t}=\mathbf{M}_{\mathcal{C}}^{-1} \underline{m}^{t}$, where $\mathcal{K}_{I}=(\mathcal{C}, \underline{m})$.

Remark 2.7. Notice that, in the statement above, $r_{i}=0$ if $Q_{i} \notin \mathcal{C}_{I}$. Moreover, the expression (1) (with non necessarily positive exponents) means that there exists a $*$-product of $I$ times ideals $\mathcal{P}_{Q_{i}}$ which is equal to a *-product of ideals $\mathcal{P}_{Q_{j}}$, which distinct factors in both sides of the equality.

If $d=2$ the situation is very simple because of Zariski's theory of complete ideals (see [70] and [71]). In this case, there exists unique factorization of complete ideals as product of simple complete ideals. Moreover, the exponents $r_{i}$ are non-negative. Lipman, in [51], provides a modern presentation of Zariski's results. The matrix $\mathbf{M}_{\mathcal{C}}$, in this case, coincides with the inverse of the transpose of the proximity matrix $\mathbf{P}_{\mathcal{C}}$ and $\left(D\left(Q_{i}\right)\right)$ is the dual $\mathbb{Z}$-basis of $\left(-E_{i}\right)$ with respect to the bilinear pairing $N^{1} \times N^{1} \rightarrow \mathbb{Z}$ given by the intersection product. The sub-semigroup $\mathcal{L}_{\mathcal{C}}$ of $\mathcal{J}_{\mathcal{C}}$ of those ideals which are $*$-products of the ideals $\mathcal{P}_{Q_{i}}$ with non-negative exponents is nothing but the free semigroup generated by the $\mathcal{P}_{Q_{i}}$. By the isomorphisms in Proposition 2.2, it corresponds to the sub-semigroup $\mathcal{L} \mathcal{G}_{\mathcal{C}}$ of $\mathcal{G}_{\mathcal{C}}$ generated by the clusters $\mathcal{K}_{\mathcal{P}_{Q_{i}}}$ and to the sub-semigroup $\mathbb{L}_{\mathcal{C}}$ of $\mathbb{E}_{\mathcal{C}}^{\sharp}$ generated by the divisors $D\left(Q_{i}\right), 0 \leq i \leq n$.

2.3. Idealistic clusters and embedded resolutions. The objective of this section is, on the one hand, to define several concepts whose aim is to describe how an effective divisor in $X$ passes through the infinitely near points involved by a cluster and, on the other hand, to state a result showing 
that, if the characteristic of the ground field $\mathbb{K}$ is 0 , then the morphism $\sigma_{\mathcal{C}}$ associated to the constellation of base points of a finitely supported ideal $I$ can be seen as the embedded resolution of a subvariety defined by general enough elements of $I$. The above mentioned concepts will help us to precise the meaning of general enough. Recall that a projective birational morphism $\pi: Z \rightarrow Y$ is an embedded resolution of a reduced subvariety $V$ of $Y$ having an isolated singularity at $Q_{0} \in X$ if $Z$ is non singular, $\pi$ induces an isomorphism of $Z \backslash \pi^{-1}\left(Q_{0}\right)$ to $X \backslash\left\{Q_{0}\right\}$ and $\pi^{-1}(V)$ is a normal crossing subscheme. Fix $\mathcal{C}=\left\{Q_{0}, \ldots, Q_{n}\right\}$ a constellation over $X$ with origin at $Q_{0}$ and set $S:=\operatorname{Spec}\left(\mathcal{O}_{X, Q_{0}}\right)$ and $S_{\mathcal{C}}:=X_{\mathcal{C}} \times_{X} S$. We shall denote also by $\sigma_{\mathcal{C}}$ to the induced morphism $S_{\mathcal{C}} \rightarrow S$. The constellation $\mathcal{C}$ can be naturally regarded as a constellation over $S$ with origin at its closed point $Q_{0}$ and $\sigma_{\mathcal{C}}=\sigma_{0} \circ \cdots \sigma_{n}: S_{\mathcal{C}}=S_{n+1} \rightarrow \cdots \rightarrow S_{1} \rightarrow S_{0}=S$ being its associated composition of blowing-ups.

Definition 2.8. Let $\mathcal{K}=(\mathcal{C}, \underline{m})$ be a cluster, with $\mathcal{C}$ as above. Let $D$ be an effective divisor on $S$.

(a) For $1 \leq i \leq n$, the divisor on $S_{i}$ given by $\check{D}_{i}:=\left(\sigma_{0} \circ \cdots \circ \sigma_{i-1}\right)^{*} D-$ $\sum_{j=0}^{i-1} m_{i} E_{i}^{*}$ is called the virtual transform of $D$ on $S_{i}$ with respect to the cluster $\mathcal{K}$. The virtual transform of $D$ on $S, \check{D}_{0}$, will be considered to be $D$.

(b) $D$ is said to pass (resp. to pass effectively) (resp. to pass properly) through $\mathcal{K}$ if for any $J=\left\{i_{1}<\cdots<i_{k}\right\}$ with $k=1$ (resp. $k=1$ ) (resp. $1 \leq k \leq d$ ) such that $E_{J}:=E_{i_{1}} \cap \cdots \cap E_{i_{k}} \subseteq S_{\mathcal{C}}$ is not empty, the multiplicity at $Q_{i_{k}}$ of the inverse image $D_{J}$ of $\check{D}_{i_{k}}$ on $E_{i_{1}} \cap \cdots \cap E_{i_{k-1}} \subseteq S_{i_{k}}\left(\right.$ or $S_{i_{k}}$ if $\left.k=1\right)$ is $\geq($ resp. $=)$ (resp. $\left.=\right)$ $m_{J}:=m_{i_{k}}$.

If $D$ and $\mathcal{K}$ are as above and $D$ passes properly with respect to $\mathcal{K}$, we denote the projective tangent cone to $D_{J}$ at $Q_{i_{k}}$ by $T C(D)_{J}$. This is a hypersurface of degree $m_{J}$ in $B_{J}:=E_{i_{1}} \cap \cdots \cap B_{i_{k}} \cong \mathbb{P}^{d-k}$.

Proposition 2.5. [9, Prop. 3.4] With the notations of Definition 2.8, the map which takes $D$ to $\check{D}_{n+1}$ (the virtual transform on $S_{n+1}=S_{\mathcal{C}}$ ) is a one to one correspondence between the set of effective divisors in $S$ which pass through $\mathcal{K}$ and the complete linear system $|-D(\mathcal{K})|$ on $S_{\mathcal{C}}$. Moreover, for any effective divisor $D$ in $S$ :

(a) $D$ passes effectively through $\mathcal{K}$ if and only if, for any $Q_{i} \in \mathcal{C}$, the multiplicity of the strict (or proper) transform of $D$ at $Q_{i}$ is $m_{i}$.

(b) If $D$ passes properly through $\mathcal{K}$ then, for any $J$ as in Definition 2.8:

(i) the subvariety $E_{J}$ on $S_{\mathcal{C}}$ is not contained in the strict transform $\tilde{D}$ of $D$, 
(ii) for $1 \leq k<d-1$, the scheme $E_{J} \cap \tilde{D}$ is the strict transform by $\sigma_{J}: E_{J} \rightarrow B_{J}$ of $T C(D)_{J}$ and for any $i \rightarrow J$ (i.e. $i \rightarrow i_{\ell}$, $1 \leq \ell \leq k)$, the multiplicity at $Q_{i}$ of the strict transform of $T C(D)_{J}$ is $m_{i}$.

Given an element $f \in R_{Q_{0}}=\mathcal{O}_{X, Q_{0}}$ we denote by $H_{f}$ the hypersurface in $S$ defined by $f$.

Definition 2.9. A $r$-uple $\left(f_{1}, \ldots, f_{r}\right)$ of elements in $R_{Q_{0}}$ with $1 \leq r<$ $d$ is said to be non degenerated with respect to a cluster $\mathcal{K}=(\mathcal{C}, \underline{m})$ if the hypersurfaces $H_{f_{1}}, \ldots, H_{f_{r}}$ pass properly through $\mathcal{K}$ and, for any $J$ such that $\operatorname{dim} E_{J} \geq 1$, the hypersurfaces $\left\{T C\left(H_{f_{i}}\right)_{J}\right\}_{i=1}^{j}$ of $B_{J}$ intersect transversally except maybe at proper points of $B_{J}$ in $\mathcal{C}$.

Proposition 2.6. [9, Prop. 3.6] If $\left(f_{1}, \ldots, f_{r}\right)$ is non degenerated with respect to $\mathcal{K}$, then $\sigma_{\mathcal{C}}: S_{\mathcal{C}} \rightarrow S$ is an embedded resolution of the subvariety of $S$ defined by $f_{1}, \ldots, f_{r}$.

Theorem 2.3. If the characteristic of the ground field $\mathbb{K}$ is 0 , I is a finitely supported ideal of $R_{Q_{0}}$ and $\mathcal{C}$ is its constellation of base points $\mathcal{C}_{I}$, then the above morphism $\sigma_{\mathcal{C}}: S_{\mathcal{C}} \rightarrow S$ is an embedded resolution of the subvariety of $S$ defined by $r, 1 \leq r<d=\operatorname{dim} X$, general elements in $I$.

Proof. It follows from the preceding proposition and the fact that, since the characteristic of $\mathbb{K}$ is 0 , a $r$-uple of general elements of $I$ is non degenerated with respect to $\mathcal{K}_{I}[9$, Prop. 3.8].

2.4. Characteristic cones and factorization properties. As we have already seen, the results in dimension 2 concerning unique factorization of complete ideals as a product of simple complete ideals do not extend to higher dimensions. The use of characteristic cones provides an interesting framework to study factorization properties of complete ideals in dimension greater than 2. The main objective of this section is to provide an overview of this fact. To begin with, we shall define some convex cones related to a projective morphism, providing also some basic properties. Afterwards, we shall consider the particular case in which such a morphism is the one associated with a constellation. Let $f: V \rightarrow Y$ be a projective morphism between algebraic schemes over $\mathbb{K}$. Denote by $N_{1}(V / Y)$ (resp. $\left.N^{1}(V / Y)\right)$ the free abelian group of 1-dimensional cycles on $V$ whose support contracts (by $f$ ) to a closed point in $Y$ (resp. Cartier divisors on $V$ ) modulo numerical equivalence. Recall that a 1-dimensional cycle $C$ (resp. a Cartier divisor $D$ ) is numerically equivalent to 0 iff $D \cdot C=0$ for all Cartier divisors $D$ (resp. all integral curves $C$ contracted to a closed point of $Y$ ) on $V$. Intersection theory provides a $\mathbb{Z}$-bilinear pairing $N^{1}(V / Y) \times N_{1}(V / Y) \rightarrow \mathbb{Z}$ which extends to a $\mathbb{R}$-bilinear pairing $A^{1}(V / Y) \times A_{1}(V / Y) \rightarrow \mathbb{R}$, where 
$A^{1}(V / Y):=N^{1}(V / Y) \otimes_{\mathbb{Z}} \mathbb{R}$ and $A_{1}(V / Y):=N_{1}(V / Y) \otimes_{\mathbb{Z}} \mathbb{R}$. The dimension $\rho(V / Y)$ of $A^{1}(V / Y)$ is finite and the above intersection pairing makes $A^{1}(V / Y)$ and $A_{1}(V / Y)$ dual vector spaces [43, Chap. IV, Sect. 4]. For simplicity of notation, given a contracted effective curve $C$ (resp. a Cartier divisor $D$ ) on $V$, its classes in $N_{1}(V / Y)$ and $A_{1}(V / Y)$ (resp. $N^{1}(V / Y)$ and $\left.A^{1}(V / Y)\right)$ will also be denoted by $C$ (resp. $\left.D\right)$. Let $N E(V / Y)$ be the cone of curves of $V$ relative to $f$, that is, the convex cone in $A_{1}(V / Y)$ generated by the classes of effective contracted curves in $V$. Denote by $P(V / Y)$ the nef cone relative to $f$ (also called semiample cone), that is, the dual cone of $N E(V / Y)$ or, equivalently, the convex cone in $A^{1}(V / Y)$ consisting of vectors $x$ such that $x \cdot C \geq 0$ for every contracted effective curve in $V$. According to [43, Chap. IV, Sect. 4], the cone $P(V / Y)^{o} \cup\{0\}$ $\left(P(V / Y)^{o}\right.$ being the topological interior of $\left.P(V / Y)\right)$ is generated by the classes of the relatively ample divisors $D$ (this means that, for every coherent sheaf $\mathcal{F}$, the canonical map $f^{*} f_{*} \mathcal{F} \otimes \mathcal{O}_{V}(m D) \rightarrow \mathcal{F} \otimes \mathcal{O}_{V}(m D)$ is surjective for all $m$ sufficiently large or, equivalently, $Y$ is covered by affine subsets $U$ such that the restriction of $D$ to $f^{-1}(U)$ is ample). The characteristic cone relative to $f, \tilde{P}(V / Y)$, is defined to be the convex cone of $A^{1}(V / Y)$ generated by the classes of Cartier divisors $D$ such that the natural sequence $f^{*} f_{*} \mathcal{O}_{V}(D) \rightarrow \mathcal{O}_{V}(D) \rightarrow 0$ is exact, The inclusion $\tilde{P}(V / Y) \subseteq P(V / Y)$ is clear. Moreover, since some multiple of an ample divisor is generated by global sections, it follows that $P(V / Y)^{\circ} \subseteq \tilde{P}(V / Y)$ and hence $P(V / Y)^{o}=\tilde{P}(V / Y)^{o}$. Notice that, since $f$ is projective, there exist relatively ample divisors and, therefore, the dimension of both cones $P(V / Y)$ and $\tilde{P}(V / Y)$ is $\rho(V / Y)$. When $Y=\operatorname{Spec}(\mathbb{K})$, the above defined spaces and convex cones are denoted by $A_{1}(V), A^{1}(V), N E(V), P(V)$ and $\tilde{P}(V)$ respectively. Assume now that $S=\operatorname{Spec}\left(\mathcal{O}_{\mathrm{X}, \mathrm{Q}_{0}}\right)$, with $X$ and $Q_{0}$ as in the preceding sections, and $\mathcal{C}=\left\{Q_{0}, \ldots, Q_{n}\right\}$ is a constellation over $S$ with associated composition of blowing-ups $\sigma_{\mathcal{C}}: S_{\mathcal{C}} \rightarrow S$. In this case $N^{1}\left(S_{\mathcal{C}} / S\right)$ coincides with the free abelian group $\mathbb{E}_{\mathcal{C}}$ and $\left\{E_{1}, \ldots, E_{n}\right\}$ is a $\mathbb{R}$-basis of $A^{1}\left(S_{\mathcal{C}} / S\right)$ [20, Lem. 15]. Moreover, the characteristic cone $\tilde{P}\left(S_{\mathcal{C}} / S\right)$ (resp. nef cone $P\left(S_{\mathcal{C}} / S\right)$ ) is the one generated by the image in $A^{1}\left(S_{\mathcal{C}} / S\right)$ of the divisors $D$ such that $-D$ (resp. $D$ ) belongs to $\mathbb{E}_{\mathcal{C}}^{\sharp}$ (resp. $\left.\operatorname{Nef}\left(S_{\mathcal{C}} / S\right)\right)$. If $d=\operatorname{dim} S=2, N^{1}\left(S_{\mathcal{C}} / S\right)$ and $N_{1}\left(S_{\mathcal{C}} / S\right)$ are identified with $N^{1}$. Taking into account the unique factorization of the ideals in $\mathcal{J}_{\mathcal{C}}$ as a product of the simple complete ideals $\mathcal{P}_{Q_{i}}$ (by Zariski's theory) and Proposition 2.2 , one has that the semigroup $\mathbb{E}_{\mathcal{C}}^{\sharp}$ is freely generated by the divisors $D\left(Q_{i}\right)$. This implies that the cone $\tilde{P}\left(S_{\mathcal{C}} / S\right)$ is the regular cone (which coincides with $P\left(S_{\mathcal{C}} / S\right)$ ) generated by the images in $A^{1}\left(S_{\mathcal{C}} / S\right)$ of the divisors $-D\left(Q_{i}\right)$. For $d>2$, the cone $\tilde{P}\left(S_{\mathcal{C}} / S\right)$ contains the regular 
sub-cone $L_{\mathcal{C}}$ generated by the divisors $-D\left(Q_{i}\right)$ but, in general, one has $L_{\mathcal{C}} \neq \tilde{P}\left(S_{\mathcal{C}} / S\right)$. If $d>2$, the cone $\tilde{P}\left(S_{\mathcal{C}} / S\right)$ is not, in general, regular (as we shall see later) and, hence, there is not, in general, unique factorization of finitely supported complete ideals as $*$-product of $*$-simple ideals. Furthermore, the regularity of the characteristic cone does not imply unique factorization of complete ideals (see [8, Example 4.2]). There is a weaker notion than the unique factorization which is detected from the structure of the characteristic cone: the semi-factoriality.

Definition 2.10. Let $G$ be a commutative semigroup with cancellation law. An element $g \in G \backslash\{0\}$ is called extremal if $g$ has no inverse in $G$ and if a factorization (additively written) $n g=a+b$ (with $n$ an integer) implies that $s a=q g$ and $t b=p g$ for suitable integers $a, b, p, q$. Two extremal elements $x$ and $y$ are called equivalent, $x \sim y$, if there are positive integers $m$ and $n$ such that $n x=m y$. $G$ is semi-factorial if to each $g \in G$ with $g \neq 0$ there is an integer $n>0$ such that $n g$ is a sum of extremal elements, and this factorization is unique in the following sense: if $n g=a_{1}+\ldots+a_{s}$, $a_{i}$ extremal, $a_{i} \nsim a_{j}$ for $i \neq j$, and $m g=b_{1}+\ldots+b_{t}, b_{i}$ extremal, $b_{i} \nsim b_{j}$ if $i \neq j$, then $s=t$ and $a_{i} \sim b_{i}$ after reindexing.

Notice that an ideal $I \in \mathcal{J}_{\mathcal{C}}$ is extremal in $\left(\mathcal{J}_{\mathcal{C}}, *\right)$ iff $D\left(\mathcal{K}_{I}\right)$ is extremal in $\mathbb{E}_{\mathcal{C}}^{\sharp}$ iff $-D\left(\mathcal{K}_{I}\right)$ generates an extremal ray of the cone $\tilde{P}\left(S_{\mathcal{C}} / S\right)$. From this fact, it can be easily deduced the following result:

Proposition 2.7. The semigroup $\mathcal{J}_{\mathcal{C}}$ is semi-factorial if and only if the cone $\tilde{P}\left(S_{\mathcal{C}} / S\right)$ is simplicial (that is, it is spanned by linearly independent elements).

The following result and the examples mentioned below show that, in general, the semi-factoriality of $\mathcal{J}_{\mathcal{C}}$ does not hold if $d>2$.

Proposition 2.8. [20, Th. 20] Suppose that $S=\operatorname{Spec}(R)$, where $R$ is the localization at $(x, y, z)$ of the polynomial ring $\mathbb{K}[x, y, z]$. Let $Q_{0}$ be the closed point of $S$ and let $\mathcal{C}_{n}=\left\{Q_{0}, Q_{1}, \ldots, Q_{n}\right\}$ be a constellation over $S$ such that $Q_{1}, \ldots, Q_{n}$ are $n$ closed points in general position on the exceptional divisor associated to the blowing-up at $Q_{0}$. Then $\tilde{P}\left(S_{\mathcal{C}_{n}} / S\right)$ is simplicial if and only if $n \leq 2$.

There are several examples in the literature showing that the characteristic cone $\tilde{P}\left(S_{\mathcal{C}} / S\right)$ can have very different shapes, indicating the existence of different factorization's phenomena:

(i) It can be polyhedral (that is, finitely generated) but not simplicial [8, Example 4.1].

(ii) It can have infinitely many extremal rays [20, Example 2]. 
(iii) It can be non-closed ([8, Example 4.3] and [20, Example 3]).

(iv) As we have pointed out before, it can be regular but with $\mathcal{J}_{\mathcal{C}}$ not having unique factorization [8, Example 4.2].

2.5. Toric constellations. Now we consider the toric constellations and proximity. We begin by recalling some definitions and fixing notations for toric varieties (for a detailed treatment see some of the basic references on this subject, e.g. chapter 1 of [59] or [42]). Let $N \cong \mathbb{Z}^{d}$ be a lattice of dimension $d \geq 2$ and $\Sigma$ a fan in $N_{\mathbb{R}}=N \otimes_{\mathbb{Z}} \mathbb{R}$, i.e. a finite set of strongly convex rational polyhedral cones such that every face of a cone of $\Sigma$ belongs to $\Sigma$ and the intersection of two cones of $\Sigma$ is a face of both. Denote by $X_{\Sigma}$ the toric variety over a field $\mathbb{K}$ associated with $\Sigma$, equipped with the action of an algebraic torus $T \cong\left(\mathbb{K}^{*}\right)^{d}$. There is a one to one canonical correspondence between the $T$-orbits in $X_{\Sigma}$ and the cones of $\Sigma$. Two basic facts of this correspondence are that the dimension of a T-orbit is equal to the codimension of the corresponding cone, and that a T-orbit is contained in the closure of another T-orbit if and only if the cone associated with the first one contains the cone associated with the second one. The morphisms of toric varieties are the equivariant maps induced by the maps of fans $\varphi:\left(N^{\prime}, \Sigma^{\prime}\right) \rightarrow(N, \Sigma)$ such that $\varphi: N^{\prime} \rightarrow N$ is a $\mathbb{Z}$-linear homomorphism whose scalar extension $\varphi: N_{\mathbb{R}}^{\prime} \rightarrow N_{\mathbb{R}}$ has the property that for each $\sigma^{\prime} \in \Sigma^{\prime}$ there exists $\sigma \in \Sigma$ such that $\varphi\left(\sigma^{\prime}\right) \subset \sigma$; (see [59], 1.5). Let $X_{0}:=X_{\Sigma_{0}} \cong \mathbb{K}^{d}$ be the $d$-dimensional affine toric variety associated with the fan $\Sigma_{0}$ formed by all the faces of a regular d-dimensional rational cone $\Delta$ in $N_{\mathbb{R}}$. Recall that a rational cone is called regular (or nonsingular) if the primitive integral extremal points form a subset of a basis of the lattice. A toric constellation of infinitely near points is a constellation $\mathcal{C}=\left\{Q_{0}, \ldots, Q_{n}\right\}$ such that each $Q_{j}$ is a fixed point for the action of the torus in the toric variety $X_{j}$ obtained by blowing-up $X_{j-1}$ with center $Q_{j-1}, 1 \leq j \leq n$. If a toric constellation is a chain, it is called a toric chain. The identification of constellations stated after definition 2.1 is the same in the toric case, with equivariant isomorphisms.

Codification of toric constellations and proximity.

By choosing a fixed ordered basis $\mathcal{B}=\left\{v_{1}, \ldots, v_{d}\right\}$ of the lattice $N$ we obtain a codification of the toric constellations, as well as criteria for proximity and (as shown in the following) linear proximity.Let $\Delta=\langle\mathcal{B}\rangle$ be the (regular) cone generated by the basis $\mathcal{B}$. The blowing-up $\sigma_{i}: X_{i} \rightarrow X_{i-1}$ of the closed orbit $Q_{i-1}$, is described as an elementary subdivision of a fan, as follows. The variety $X_{1}$ is the toric variety associated with the fan $\Sigma_{1}$, obtained as the minimal subdivision of $\Sigma_{0}$ which contains the ray through 
$u=\sum_{1 \leq j \leq d} v_{j}$.

For each integer $i, 1 \leq i \leq d$, let $\mathcal{B}_{i}$ be the ordered basis of $N$ obtained by replacing $v_{i}$ by $u$ in the basis $\mathcal{B}$; and let $\Delta_{i}:=\left\langle\mathcal{B}_{i}\right\rangle$. The exceptional divisor $B_{0}$ is the closure in $X_{1}$ of the $T$-orbit defined by the ray through $u$, and each T-fixed point in $X_{1}$ corresponds to a maximal cone $\Delta_{i}$ of the fan $\Sigma_{1}, 1 \leq i \leq d$. The choice of the point $Q_{1} \geq Q_{0}$ is thus equivalent to the choice of an integer $a_{1}, 1 \leq a_{1} \leq d$, which determines a cone $\Delta_{a_{1}}$ of the fan $\Sigma_{1}$. The subdivision $\Sigma_{2}$ of $\Sigma_{1}$ corresponding to the blowing-up of $Q_{1}$ is obtained by replacing $\Delta_{a_{1}}$ (and its faces) in $\Sigma_{1}$ by the cones $\Delta_{a_{1} i}:=\left\langle\mathcal{B}_{a_{1} i}\right\rangle$ (and their faces), where $\mathcal{B}_{a_{1} i}$ is the ordered basis of $N$ obtained from $\mathcal{B}_{a_{1}}$ by the substitution of its $i$-th vector by $\sum_{v \in \mathcal{B}_{a_{1}}} v$. The choice of $Q_{2} \in B_{1}$ is equivalent to the choice of an integer $a_{2}, 1 \leq a_{2} \leq d$, which determines a (regular) cone $\Delta_{a_{1} a_{2}}$.Proceeding by induction on $n$ we obtain a codification of toric chains and also constellations, since for each $Q \in \mathcal{C}$, the constellation $\mathcal{C}^{Q}$ is a chain. The codification is given by trees with weighted edges, where the weights are integers $a, 1 \leq a \leq d$, which give the direction in which the following blowing-up is done. The precise description follows.

Definition 2.11. Let $\Gamma$ be a tree, $\mathcal{E}(\Gamma)$ the set of edges of $\Gamma, \mathrm{d}$ an integer, $\mathrm{d} \geq 2$.

A $d$-weighting of $\Gamma$ is a map $\alpha: \mathcal{E}(\Gamma) \rightarrow\{1, \ldots, \mathrm{d}\}$ which associates to each edge of $\Gamma$ a positive integer not greater than $d$, such that two edges with a common origin have different weights. A couple $(\Gamma, \alpha)$ is called a d-weighted tree.

Proposition 2.9. Let $\mathcal{B}$ be an ordered basis of the lattice $N$ and $n$ a positive integer.

(a) The map which associates to each sequence of integers $\left\{a_{1}, \ldots, a_{n}\right\}$ such that $1 \leq a_{i} \leq d, 1 \leq i \leq n$, the toric chain $\left\{Q_{0}, \ldots, Q_{n}\right\}$ where $Q_{0}$ is the T-orbit corresponding to the cone $\Delta=\langle\mathcal{B}\rangle$, and where $Q_{i}$, $1 \leq i \leq n$, is the T-orbit in $X_{i}$ corresponding to the cone $\Delta_{a_{1} \ldots a_{i}}$ of the fan $\Sigma_{i}$, is a bijection between the set of such sequences and the set of d-dimensional toric chains with $n+1$ points.

(b) A natural bijection between the set of d-dimensional toric constellations and the set of d-weighted trees is induced by the correspondence (a).

Remark 2.8. Note that in a d-weighted tree each vertex is the origin of at most $\mathrm{d}$ edges. A d-weighting of a tree $\Gamma$ induces a partition of the set $\mathcal{E}(\Gamma)$ of edges, where two edges are in the same class if they have the same weight. To each class of isomorphism of d-dimensional toric constellations is associated a unique class of isomorphism of trees equipped with a partition of the set of edges, partition with at most d classes of edges [34]. 
Given a toric constellation by a d-weighted graph, a vertex following $q$ through a chain with edges weighted by a sequence $\left(a_{1}, \ldots, a_{k}\right)$ is denoted by $q\left(a_{1}, \ldots, a_{k}\right)$; if $Q$ is the point corresponding to $q$, then the point corresponding to $q\left(a_{1}, \ldots, a_{k}\right)$ is written in a similar way $Q\left(a_{1}, \ldots, a_{k}\right)$.

Proposition 2.10. (Criterion for proximity in terms of a codification) $Q\left(a_{1}, \ldots, a_{k}\right) \rightarrow Q$ if and only if $a_{1} \neq a_{j}$ for $2 \leq j \leq k$.

Proof. The criterion follows from the fact that this is the condition to obtain, by elementary subdivisions of a regular fan, an adjacent maximal cone $\Delta_{a_{1} \ldots a_{k}}$ (corresponding to a 0 -dimensional orbit) to the central ray of $\Delta_{a_{1}}$ (corresponding to the exceptional divisor) of the first subdivision of the cone $\Delta$ corresponding to $Q$. This is equivalent to saying that $Q\left(a_{1}, \ldots, a_{k}\right) \in E_{Q}$, i.e. $Q\left(a_{1}, \ldots, a_{k}\right) \rightarrow Q$.

We obtain a characterization of toric P-Enriques diagrams and the minimum dimension for a toric constellation with a given P-Enriques diagram ( [34], [35])

Theorem 2.4. A P-Enriques diagram $(\Gamma,(\sim))$ is toric, i.e. may be induced by a toric constellation, if and only if:

(a) The proximity index is non-decreasing, i.e. ind $(r) \geq \operatorname{ind}(q)$ if $r \succeq q$.

(b) If $r$ is proximate to $q$, then there is at most one vertex $s$ consecutive to $r$ and not proximate to $q$, i.e. if $r \leadsto q$ then $\#\left\{s \in r^{+} \mid s \chi_{\rightarrow}\right.$ $q\} \leq 1$.

If these conditions hold, then the minimum dimension $d t_{\mathcal{P}}(\Gamma,(\sim))$ of a toric constellation inducing the given P-Enriques diagram $(\Gamma,(\sim))$ is $\max \left(2, \max _{q \in \Gamma}(\operatorname{ind}(q)+s(q))\right)$, where $s(q):=\#\left\{r \in q^{+} \mid \operatorname{ind}(r)>\operatorname{ind}(q)\right\}$ is the number of consecutive points to $q$ whose proximity index is greater than the proximity index of $q$.

Remark 2.9. The minimum dimension $d t_{\mathcal{P}}$ may be greater than $d_{\mathcal{P}}$, the dimension in the not necessarily toric case (Theorem 2.1), because there are less points available, so one needs to add $s(q)$ to the proximity index, not just 1 as in the general case.

Corollary 2.1. A P-Enriques diagram $(\Gamma,(\sim))$ whose graph $\Gamma$ is a chain, is toric if and only if the proximity index is not decreasing. In this case, the minimum dimension of an associated constellation is the index of the terminal point (and at least 2).

Proof. In the toric chain case the condition (b) of the theorem is automatically satisfied and $\left.\max _{q \in \Gamma}(\operatorname{ind}(q)+s(q))\right)=\max _{q \in \Gamma}(\operatorname{ind}(q))$ holds. 


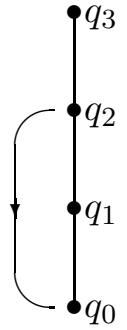

Figure (1)

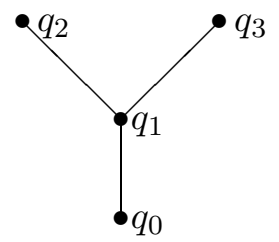

Figure (2).

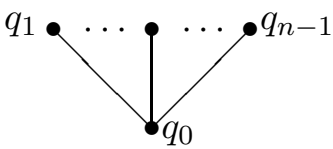

Figure (3).

Examples 2.1. (1) The simplest example of a non-toric P-Enriques diagram is a chain with four vertices, say $q_{0}, q_{1}, q_{2}, q_{3}$ such that, besides the trivial proximities of consecutive vertices, the only other proximity is $q_{2} \rightarrow q_{0}$. In this example one has $\operatorname{ind}\left(q_{2}\right)=2$ and $\operatorname{ind}\left(q_{3}\right)=1$; condition (a) fails.

(2) Another example of a non-toric case is a graph of type $\mathbb{D}_{4}$, with a non-central vertex as the root, and with only the proximities of consecutive vertices. In this case condition (b) fails. Remark that both cases may be induced by two dimensional constellations.

(3) If the central vertex is the root in a graph of type $\mathbb{D}_{n}$, with $n \geq 4$, and if the only proximities are those of consecutive vertices, then conditions (a) and (b) hold; the minimal dimension of a constellation inducing this P-Enriques diagram is $n-1$ for toric constellations and two for non-toric ones. If $q_{0}$ is the root, then $\operatorname{ind}\left(q_{0}\right)=0, s\left(q_{0}\right)=n-1, t\left(q_{0}\right)=1$, and $\operatorname{ind}(q)=1, s(q)=0, t(q)=0$ for each $q \neq q_{0}$.

(See Figures (1), (2) and (3)).

\section{Linear proximity and characteristic cones}

In dimension two the exceptional divisors appearing in the definition of the proximity relations are (rational) curves. In higher dimension we introduce, in the toric case, a condition involving curves which will be finer, in general, than the proximity. This new condition arises naturally for toric clusters in higher dimension, from the generalization of the proximity inequalities. 
Definition 2.12. Let $\mathcal{C}=\left\{Q_{0}, \ldots, Q_{n}\right\}$ be a toric constellation. A point $Q_{j}$ is linear proximate to a point $Q_{i}$ with respect to a one dimensional Torbit $\ell \subset B_{i}$ if $Q_{j}$ belongs to the strict transform in $X_{j}$ of the closure of $\ell$. This relation is denoted by $Q_{j} \rightarrow Q_{i}$, or $Q_{j} \stackrel{\ell}{\rightarrow} Q_{i}$ if we need to specify the line $\ell$ involved.

If $R \rightarrow Q$ then $R \rightarrow Q$, but the converse does not hold in general.

Proposition 2.11. (Criterion for linear proximity) Let $Q$ be a point in a toric constellation of dimension $d$. Each 1-dimensional orbit $\ell$ in the exceptional divisor $B_{Q}$ contains in its closure only two fixed points, say $Q(a)$ and $Q(b)$. Then $R \rightarrow Q$ if and only if there are integers $a, b$ and $m$ such that $a \neq b, 1 \leq a \leq d, 1 \leq b \leq d, 0 \leq m$ and $R=Q\left(a, b^{[m]}\right)$ or $R=Q\left(b, a^{[m]}\right)$, where $x^{[m]}$ means $x$ repeated $m$ times.

Proof. The wall running between the cones corresponding to $Q(a)$ and $Q(b)$ is the cone corresponding to the line defined by this two points in $B_{Q}$. The only maximal cones, obtained by elementary subdivisions, having this wall as a face are those corresponding to the points $Q\left(a, b^{[m]}\right)$ or $Q\left(b, a^{[m]}\right)$ for some $m \geq 0$.

In dimension two, proximity and linear proximity are equivalent. One implication may be generalized for toric chains in any dimension.

Proposition 2.12. If $\mathcal{C}$ is a toric chain (in any dimension), the proximity relation determines the linear proximity relation.

Proof. If $R \rightarrow Q$, then $P \rightarrow Q$ for any $P$ such that $R \geq P \geq Q, P \neq Q$, and these are the only proximities, for the intermediate points in the chain from $Q$ to $R$, besides the proximities of consecutive points. Conversely, assuming this property, then $R \stackrel{\ell}{\rightarrow} Q$ for the line $\ell$ determined by the point $Q^{+}$and the direction $Q^{++}$in the projective space $B_{Q}$, if $Q^{++}$is defined and precedes $R$, or any line through $Q$ otherwise. Indeed, this assumption forces the code of $R$ to be $Q\left(a, b^{[m]}\right)$ for some weights $a$ and $b, m \geq 0$.

Note that in general the linear proximity does not determine the proximity, even for chains.

\section{LP-Enriques diagrams.}

We introduce now some definitions leading to the notion of the so called (linear proximity) LP-Enriques diagrams. This is a LP generalization, for toric constellations of dimension higher than two, of the Enriques diagrams of two dimensional constellations. We will give later an application of these 
diagrams to prove a converse Zariski theorem. Given a rooted tree $\Gamma$, a sub graph formed by two chains with a common root and no common edge is called a bi-chain. If $\Gamma$ is the rooted tree associated with a toric constellation $\mathcal{C}, q$ the vertex corresponding to $Q \in \mathcal{C}$ and $\ell$ is a 1 -dimensional orbit in $B_{Q}$, then $\Gamma_{q}(\ell)$ denotes the full subgraph of $\Gamma$ with vertices corresponding to $\mathrm{Q}$ and to the points $R \in \mathcal{C}$ such that $R \rightarrow Q$. Let $\Gamma(q)$ be the family of the maximal $\Gamma_{q}(\ell)$ when $\ell$ describes the set of one dimensional orbits in $B_{Q}$. A vertex $q \in \Gamma$ is called simple (resp. ramified) if $\left|q^{+}\right|=1$ (resp. if $\left|q^{+}\right|>1$ ). The following properties are easily checked with the linear proximity criterion (Proposition 2.11).

Proposition 2.13. Let $\mathcal{C}$ be a toric constellation, $\Gamma$ the associated tree.

1. (a) For each $q \in \Gamma$, the family $\Gamma(q)$ is non-empty and the elements of $\Gamma(q)$ are chains or bi-chains with root $q$.

(b) If $\gamma, \gamma^{\prime} \in \Gamma(q)$ and $\gamma \subset \gamma^{\prime}$, then $\gamma=\gamma^{\prime}$.

2. (a) Two distinct elements of $\bigcup_{q} \Gamma(q)$ have at most one common edge.

(b) Two edges with common ramification root vertex $q$ (resp. the edge with the simple root vertex q) belong (resp. belongs) to one and only one element of $\Gamma(q)$.

3. (a) For each $q \in \Gamma$ and $r \in q^{+}$there is at most one vertex $s \in r^{+}$ such that the chain $(q, r, s)$ is not contained in any element of $\Gamma(q)$.

(b) If $(p, \ldots, q, r)$ is a chain contained in a $\gamma \in \Gamma(p)$ and $s \in r^{+}$ satisfies 3.(a), then the chain $(p, \ldots, q, r, s)$ is contained in $\gamma$.

Definition 2.13. The LP-Enriques diagram of a toric constellation $\mathcal{C}$ is the associated graph $\Gamma_{\mathcal{C}}$ equipped with the linear proximity structure formed by the family of full subgraphs $\left\{\Gamma_{\mathcal{C}}(q) \mid q \in \Gamma_{\mathcal{C}}\right\}$.

We obtain a characterization of LP-Enriques diagrams and the minimum dimension for a toric constellation with a given LP-Enriques diagram ( [34], [35]).

Theorem 2.5. The couple $(\Gamma,\{\Gamma(q) \mid q \in \Gamma\})$, given by a tree $\Gamma$ and a family of full subgraphs $\Gamma(q)$, is the LP-Enriques diagram of a toric constellation $\mathcal{C}$ if and only if the properties 1, 2 and 3 hold. The minimum dimension of the constellations with given LP-Enriques diagram is $d_{\mathcal{P} \mathcal{L}}=$ $\max \left(2, \max _{q \in \Gamma}\left(\left|q^{+}\right|+n_{q}\right)\right)$, where $n_{q}=\max _{r \in q^{+}} \#\{\gamma \in \Gamma(q) \mid r \in \gamma$ and $\gamma$ is a chain of length $>1\}$

Remark 2.10. A LP-Enriques diagram may be induced by two non-isomorphic constellations. In some cases, for instance if for each vertex $q$ the family $\Gamma(q)$ has only bi-chains or is reduced to the vertex, then the 
constellation inducing the given LP-Enriques diagram is unique (up to isomorphism of constellations), and its dimension is $\left|q_{0}^{+}\right|$if $q_{0}$ denotes the root. The maximum possible linear proximity dimension $d_{L P}$ of a fixed tree, by changing its LP structure, is the number of edges. In this case all the chains (resp. bi-chains) have only one edge (resp. two edges) or are reduced to a vertex, for the maximal ones.

Characteristic cones of toric constellations.

For toric constellations the characteristic cone may be explicitly obtained (see [9], theorem 2.10). Note that in this case the characteristic cone coincides with the semiample cone (see [42], page 47). The natural ideals to consider are the invariant ideals for the toric action, so that the constellations of base points are toric. The conditions that such an ideal $\mathcal{I}$ is finitely supported and complete are formulated in terms of the Newton polyhedron $\mathcal{N}$ of $\mathcal{I}$ relative to the local system of parameters of the local ring, induced by a basis of the lattice where the fan lives. The first condition is that the fan associated to the Newton polyhedron (which gives the normalized blowing-up of center $\mathcal{I}$ ) admits a regular subdivision obtained by elementary subdivisions of the regular cone $\Delta$ corresponding to $Q_{0}$; and the second one is that every monomial corresponding to an integral point of $\mathcal{N}+\Delta^{\vee}$ is in $\mathcal{I}$, where $\Delta^{\vee}$ denotes the dual cone of $\Delta$. The following result generalizes, for toric constellations in any dimension, the two dimensional proximity inequalities found by Enriques. Recall Proposition 2.11.

Theorem 2.6. Let $\mathcal{C}$ be a toric constellation of dimension $d$.

The characteristic cone associated with $\mathcal{C}$ is the cone generated by the classes of the divisors $D_{\underline{m}}=\sum_{Q \in \mathcal{C}} m_{Q} E_{Q}^{*} \quad$ such that $\underline{m}$ verifies the linear proximity inequalities $m_{Q} \geq \sum_{P \stackrel{\ell}{\rightarrow} Q} m_{P} \quad$ for each $Q \in \mathcal{C}$ and each $\ell=\ell(Q(a), Q(b)), a \neq b 1 \leq a \leq d, 1 \leq b \leq d$.

Proof. The linear proximity inequalities are necessary, since they are equivalent to $\left(D_{\underline{m}} \cdot \bar{\ell}\right) \leq 0$ for a semiample divisor $-D_{\underline{m}}$ and the closure $\bar{\ell}$ of each one dimensional orbit $\ell(Q(a), Q(b))$. Conversely, if these inequalities hold, then $-D_{m}$ is semiample since the classes of the closures of the one dimensional orbits generate the cone of the numerically effective curves $N E$, and then the divisor is $\sigma$-generated because $\sigma$ is a toric morphism.

\section{Remark 2.11.}

(1) A constructive proof giving the Newton polyhedron of the unique complete ideal associated to such a divisor $D_{\underline{m}}$ (or the corresponding idealistic cluster) is presented in [9] theorem 2.10 (ii). 
(2) From Theorem 2.6 and Proposition 2.4 one can characterize, in numerical terms, which toric clusters correspond to adjoint ideals of finitely supported ideals. In fact, such toric clusters $\left(\mathcal{C}, \underline{m}^{\prime}\right)$ are exactly those such that the toric cluster given by $(\mathcal{C}, \underline{m})$, where $m_{Q}=m_{Q}^{\prime}+(d-1)$, satisfies the conditions of Theorem 2.6.

(3) Theorem 2.6 has been recently used by A. Lemahieu and W. Veys in [46] to describe the zeta functions for non degenerated hypersurfaces with respect to 3 -dimensional toric clusters and prove the monodromy conjecture for them.

Corollary 2.2. We keep the notations of the theorem. Let $\mathcal{C}=\left\{Q_{0}, \ldots, Q_{n}\right\}$ be a toric chain.

(a) The characteristic cone associated with $\mathcal{C}$ is given by

$$
m_{i} \geq \sum_{j \rightarrow i} m_{j}, 0 \leq i \leq n .
$$

(b) The divisor $D_{n}=\sum_{0 \leq i \leq n} m_{i, n} E_{i}^{*}$ associated to the special $*$-simple ideal $\mathcal{P}_{Q_{n}}$ is given by $m_{n, n}=1, m_{i, n}=\sum_{j \rightarrow i} m_{j, n}$, for $0 \leq i \leq n$.

Proof. (a) follows from the Theorem and the fact that for each point there is only one relevant inequality, since $\mathcal{C}$ is a chain. (b) follows from $(a)$ since the minimality property of $\underline{m}$ is obtained if $m_{n, n}=1$ and if every inequality involving an index $i \neq n$ becomes an equality.

The special $*$-simple ideals, and the exponents of the factorizations are determined by the linear proximities:

Theorem 2.7. Let $\mathcal{C}$ be a toric constellation.

(a) Let $\left(D_{Q}\right)_{Q \in \mathcal{C}}$ be the basis of $N^{1}$ corresponding to the special $*$-simple ideals with base points in $\mathcal{C}$. Then $D_{Q}=\sum_{P \in \mathcal{C}} m_{P Q} E_{P}^{*}$, where

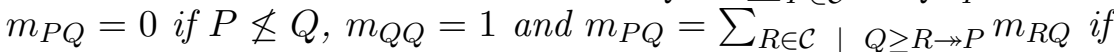
$P \leq Q$.

(b) Let $\mathbb{P}_{L}=\left(\left(l_{P Q}\right)\right)$ be the linear proximity matrix defined by $l_{P P}=1$, $l_{P Q}=-1$ if $P \rightarrow Q$ and 0 otherwise. Then ${ }^{t} \mathbb{P}_{L}$ is the basis change matrix from $\left(E_{Q}^{*}\right)$ to $\left(D_{Q}\right)$.

(c) Let $\mathcal{I}$ be a toric finitely generated ideal with base points in $\mathcal{C}$. Then the exponents of its factorization in terms of special $*$-simple ideals are: $r_{Q}=m_{Q}-\sum_{P \rightarrow Q} m_{P}$.

Proof. (a) follows from corollary 2.2, (b). (b) and (c) follow from $(a)$ and linear algebra.

Recall the definition of the LP structure of the tree $\Gamma$ associated with $\mathcal{C}$ (Proposition 2.13). 
Corollary 2.3. Let $P_{\mathcal{C}}=P\left(X_{\mathcal{C}} / X\right)$ be the characteristic cone associated with $\mathcal{C}$. The following conditions are equivalent: $(a)$ The cone $P_{\mathcal{C}}$ is regular. (b) $\left(D_{Q}\right)_{Q \in \mathcal{C}}$ is a basis of the semigroup $P_{\mathcal{C}} \cap N^{1}$. (c) The cone $P_{\mathcal{C}}$ is simplicial. (d) The special *-simple factorizations have only non negative exponents. (e) For each $Q \in \mathcal{C}$ there is only one (maximal) chain or bichain in $\Gamma(q)$.

Proof. The conditions $(a),(b),(c)$ and $(d)$ are equivalent since the divisors $D_{Q}$ form a basis of $N^{1}$. The equivalence between $(e)$ and $(c)$ follows from the preceding theorem, and the fact that the supporting hyperplanes of the maximal faces of the cone $P_{\mathcal{C}}$ are those associated with the maximal elements of $\Gamma_{Q}$ for each $Q \in \mathcal{C}$.

Remark 2.12. In particular, every toric chain constellation in any dimension has a regular characteristic cone. There are also non-chain constellations with this property.

We give now an application of the LP-Enriques diagrams for a converse Zariski Theorem for toric constellations. Recall the notations and results on the minimal LP-dimension $d_{\mathcal{L P}}$ of a LP-Enriques diagram (Theorem 2.5).

Theorem 2.8. The characteristic cone of a toric constellation is regular if and only if its LP-Enriques diagram is induced by a two dimensional constellation.

Proof. The characteristic cone of any two dimensional constellation is regular, by Zariski. Conversely, assume that the characteristic cone is regular. Then $\Gamma(q)$ has only one element for each $q \in \Gamma$, by the last Corollary. It follows necessarily that $0 \leq\left|q^{+}\right| \leq 2$. Now, $0 \leq\left|q^{+}\right| \leq 1$ implies that $0 \leq n_{q} \leq 1$ and $\left|q^{+}\right|=2$ implies that $n_{q}=0$. It follows that the minimal dimension $d_{\mathcal{L P}}$ of a constellation inducing the given LP-Enriques diagram is two.

In the general toric case, the characteristic cone $P_{\mathcal{C}}$ contains the regular sub-cone $L_{\mathcal{C}}$ which is given by the inequalities $m_{Q} \geq \sum_{P \rightarrow Q} m_{P}$ for $Q \in \mathcal{C}$. Notice that conditions in Corollary 2.3 are also equivalent to the equalities $\mathbb{L}_{\mathcal{C}}=\mathbb{E}_{\mathcal{C}}^{\sharp}$ or $L_{\mathcal{C}}=P_{\mathcal{C}}$. For a non toric two dimensional constellation $\mathcal{C}$, Zariski's theory shows that one also has $\mathbb{L}_{\mathcal{C}}=\mathbb{E}_{\mathcal{C}}^{\sharp}$ and that $L_{\mathcal{C}}=P\left(X_{\mathcal{C}} / X\right)=\tilde{P}\left(X_{\mathcal{C}} / X\right)$ is a regular cone. For $d=2$, Zariski's theory asserts also further properties as the following ones:

(i) If $I \in \mathcal{J}_{\mathcal{C}}$ and $Q \in \mathcal{C}$, then the weak transform $I_{Q}$ is a complete ideal; so $I_{Q} \in \mathcal{J}_{\mathcal{C}^{Q}}$.

(ii) If $I, J \in \mathcal{J}_{\mathcal{C}}$ then $I * J=I J$, so $I J$ is a complete ideal. 
(iii) If $\mathcal{K} \in \mathcal{G}_{\mathcal{C}}$ is given by $\mathcal{K}=(\mathcal{C}, \underline{m})$ then $\mu\left(I_{\mathcal{K}}\right)=m_{0}+1$, where $\mu$ stands for the minimal number of generators of an ideal.

The following result, due to E. Tostón [68], shows that above properties are also true for toric clusters in the sub-semigroup $\mathcal{L G}_{\mathcal{C}}$ of the galaxy $\mathcal{G}_{\mathcal{C}}$ (recall the notations used at the end of Section 2.2).

Theorem 2.9. [68, Th. 3.1, Prop 3.2, 3.4] Let $\mathcal{C}$ be a toric constellation. Then one has:

(i) If $I \in \mathcal{L}_{\mathcal{C}}$ and $Q \in \mathcal{C}$ then the weak transform $I_{Q}$ is a complete ideal and $I_{Q} \in \mathcal{L}_{\mathcal{C}_{Q}} \subseteq \mathcal{G}_{\mathcal{C} Q}$

(ii) If $I, J \in \mathcal{L}_{\mathcal{C}}$ then $I * J=I J$; so IJ is a complete ideal.

(iii) If $\mathcal{K} \in \mathcal{L} \mathcal{G}_{\mathcal{C}}$ is given by $\mathcal{K}=(\mathcal{C}, \underline{m})$ then one has $\mu\left(I_{\mathcal{K}}\right)=\left(\begin{array}{c}m_{0}+d-1 \\ d-1\end{array}\right)$.

Part (iii) follows from (ii) and the fact that, in the toric case, the conditions of effective passage through an idealistic cluster are linearly independent. For it, (ii) is applied to the ideals $I_{\mathcal{K}}$ and $M, M$ being the maximal ideal.

\section{Remark 2.13.}

(1) If $\mathcal{C}$ satisfies the equivalent conditions in Corollary 2.3 the statement of Theorem 2.9 is true for the whole galaxy of $\mathcal{C}$, as one has $\mathcal{G}_{\mathcal{C}}=$ $\mathcal{L} \mathcal{G}_{\mathcal{C}}$ and $\mathcal{J}_{\mathcal{C}}=\mathcal{L}_{\mathcal{C}}$. Thus, Zariski's theory is fully extended for such constellations. In particular, it is true for toric chains. This gives an additional insight to Theorem 2.8 .

(2) If $\mathcal{C}$ does not satisfy conditions in Corollary 2.3 then the statement of Theorem 2.9 is not longer true for ideals in $\mathcal{G}_{\mathcal{C}} \backslash \mathcal{L}_{\mathcal{C}}$ as the following examples, also due to E. Tostón in [68], show.

Examples 2.2. Consider $d=3$, coordinates $x, y, z$ and let $\mathcal{C}$ be the toric constellation consisting of two chains of respective edge weights given by $\{1,2,2\}$ and $\{3,2,1\}$. Let $\mathcal{K}$ be the cluster supported on $\mathcal{C}$ with $\underline{m}$-weights given by $3,1,1,1$ and $3,2,1,1$ respectively on above chains. Then one has

$$
I=I_{\mathcal{K}}=\left\langle x^{4}, x^{3} y, x^{2} z, x^{2} y^{2}, x^{2} y z, x y^{3}, x y^{2} z, x z^{3}, y^{3}, y^{2} z^{2}, y z^{3}, z^{5}\right\rangle .
$$

Now, if $Q$ is a 0 -dimensional $T$-orbit connected to $Q_{0}$ by the edge of weight 3 , then one has $\left(\frac{x}{z} \frac{y}{z}\right)^{2} \notin I_{Q}$ but $\left(\frac{x}{z} \frac{y}{z}\right)^{2} \in \bar{I}_{Q}$. Thus (i) is not true for $I$. On the other hand, one has $x y^{2} z \in I * M$ but $x y^{2} z \notin I M$, which shows that (ii) is not true for the ideals $I$ and $M$. Finally, $\mu(I)=\operatorname{dim}(I / I M)>$ $\operatorname{dim}(I / I * M)=\left(\begin{array}{c}3+2 \\ 2\end{array}\right)=10$, so (iii) is again not true for $I$. Notice that $\mathcal{K}$ is an idealistic cluster. However $\mathcal{K}$ is not in $\mathcal{L G}_{\mathcal{C}}$ because

$$
3=m_{Q_{0}}<\sum_{P \rightarrow Q_{0}} m_{P}=2+1+1+1+1=6 .
$$


Even it is not true that $I^{2}$ is complete when $I$ is a toric finitely supported complete ideal. This happens, for instance, if $d=4$ and $I=I_{\mathcal{K}^{\prime}}$, where $\mathcal{K}^{\prime}=$ $\left(\mathcal{C}^{\prime}, \underline{m}\right)$ is given by the constellation $\mathcal{C}^{\prime}$ consisting of the three chains with edge weights $\{1,2,3\},\{3,2,1\},\{4,2,2\}$ and respective $\underline{m}$-weights given by $3,1,1,1 ; 3,2,1,1 ; 3,1,1,1$. If $x, y, z, w$ are the coordinates, one has $x^{2} y^{2} z w \notin I^{2}$ but $x^{2} y^{2} z w \in \bar{I}^{2}$.

Remark 2.14. The exact conditions under which the equality $\mu\left(I_{\mathcal{K}}\right)=$ $\left(\begin{array}{c}m_{0}+d-1 \\ d-1\end{array}\right)$ is true for clusters are investigated in homological terms in [19].

\section{Global theory}

\subsection{Configurations, global clusters and linear systems.}

Definition 3.1. A configuration of infinitely near points (configuration, in short) is a finite union of constellations whose origins are closed points of $X$.

If $\mathcal{C}$ is a configuration, as in the case of constellations, $\sigma_{\mathcal{C}}: X_{\mathcal{C}} \rightarrow X$ will denote the composition of the blowing-ups of all the points in $\mathcal{C}$; moreover two configurations $\mathcal{C}$ and $\mathcal{C}^{\prime}$ over $X$ are identified if there exist an automorphism $\pi$ of $X$ and an isomorphism $\pi^{\prime}: X_{\mathcal{C}} \rightarrow X_{\mathcal{C}^{\prime}}$ such that $\sigma_{\mathcal{C}^{\prime}} \circ \pi^{\prime}=\pi \circ \sigma_{\mathcal{C}}$. Given a configuration $\mathcal{C}$, the relation $\geq$ and the proximity relation $\rightarrow$ between points of $\mathcal{C}$ are defined as in the case of constellations. Also, for a point $Q$ in $\mathcal{C}$, we define the concepts of associated constellation $\mathcal{C}^{Q}$ of preceding points, level of $Q$, its proximity index ind $(Q)$ and the set of consecutive points $Q^{+}$as those referred to the maximal constellation contained in $\mathcal{C}$ to which $Q$ belongs. The exceptional divisors, its strict and total transforms and the lattice of divisorial cycles with exceptional support in $X_{\mathcal{C}}$ will be denoted as in the case of constellations. Also, the proximity matrix is defined, in the same way, as the basis change matrix from the $E_{i}$ 's to the $E_{j}^{*}$ 's. If $\mathcal{C}=\bigcup_{i=1}^{r} \mathcal{C}_{i}$, where the $\mathcal{C}_{i}$ 's are disjoint constellations, we define the $P$-Enriques diagram associated to $\mathcal{C}$ as the disjoint union of the P-Enriques diagrams $\Gamma_{\mathcal{C}_{1}}, \ldots, \Gamma_{\mathcal{C}_{r}}$. We also adapt in the obvious way the concept of cluster to the global case defining it as a pair $(\mathcal{C}, \underline{m})$, where $\mathcal{C}=\left\{Q_{i}\right\}_{i=0}^{n}$ is a configuration and $\underline{m}=\left(m_{0}, \ldots, m_{n}\right)$ is a sequence of integers; also, $m_{i}$ is called the weight or virtual multiplicity of $Q_{i}$ in the cluster. As in the local case, we can associate to the cluster the divisor with exceptional support $D(\mathcal{K}):=\sum_{i=0}^{n} m_{i} E_{i}^{*}$.

Definition 3.2. A sheaf of ideals $\mathcal{I}$ on $X$ is said to be finitely supported if there exists a finite set $S$ of closed points of $X$ such that, for each closed point $Q \in X$, the stalk $\mathcal{I}_{Q}$ is a finitely supported ideal in $\mathcal{O}_{X, Q}$ (resp. $\mathcal{I}_{Q}=\mathcal{O}_{X, Q}$ ) whenever $Q \in S$ (resp. $Q \notin S$ ). This implies that there 
exists a configuration $\mathcal{C}$ over $X$ (whose roots are the points in $S$ ) such that $\mathcal{I O}_{X_{\mathcal{C}}}$ is an invertible sheaf. The configuration of base points of $\mathcal{I}$ (denoted by $\mathcal{C}_{\mathcal{I}}$ ) is the configuration with the minimal number of points having this property, that is, $\mathcal{C}_{\mathcal{I}}=\bigcup_{Q \in S} \mathcal{C}_{\mathcal{I}_{Q}}$.

Given a finitely supported ideal sheaf $\mathcal{I}$, its cluster of base points $\mathcal{K}_{\mathcal{I}}$ will be the cluster $\left(\mathcal{C}_{\mathcal{I}}, \underline{m}\right)$ where $\mathcal{C}_{\mathcal{I}}=\left\{Q_{0}, \ldots, Q_{n}\right\}$ is the above defined configuration of base points and, for $0 \leq i \leq n, m_{i}$ is the weight of $Q_{i}$ in the cluster $\mathcal{K}_{\mathcal{I}_{O}}, O \in X$ being the image of $Q_{i}$ by $\sigma_{\mathcal{C}_{\mathcal{I}}}$. Notice that Definition 2.8 makes sense also for configurations. Also, given a cluster $\mathcal{K}=(\mathcal{C}, \underline{m})$ and given a non-empty complete linear system $|R|$ on $X$, the set of elements of $|R|$ passing through $\mathcal{K}$ form a linear system $\mathcal{L}_{|R|}(\mathcal{K})$ on $X$ whose elements are in one to one correspondence with the complete linear system on $X_{\mathcal{C}}$ given by $\left|\sigma_{\mathcal{C}}^{*} R-D(\mathcal{K})\right|$. The correspondence is given by the assignation, to each effective divisor $D \in|R|$, of its virtual transform on $X_{\mathcal{C}}$ (defined in the same way as in Definition 2.8). Also, fixed a non-empty linear system $\varrho \subseteq|R|$, one can consider the ideal sheaf $\mathcal{I}(\varrho)$ whose stalks at the points of $X$ are generated by the local equations of the divisors in $\varrho$. This ideal sheaf defines a closed sub-scheme of $X$, called the base point scheme of $\varrho$. Notice that $\mathcal{I}(\varrho)$ may not be finitely supported (resp. complete). The determination of the dimension of a linear system of hypersurfaces passing through a cluster is a very classical problem in algebraic geometry, mainly considered when the configuration $\mathcal{C}$ consists of a set of (proper) points on a projective space $\mathbb{P}^{r}$ in general position. It has been present in the works of Bézout, Plücker, Cremona, M. Noether, Bertini, C. Segre, Castelnuovo, Enriques, Severi and, more recently, Alexander, Hirschowitz, Ciliberto, Miranda, Harbourne, among many others. There are several conjectures and open questions on this problem and it is related to other topics like the 14-th problem of Hilbert [58], the symplectic packing problem [55, 69] or the Waring's problem in number theory (see Section 7 of [16]). For interesting surveys on this subject we refer the reader to [32], [16], [56] or [37]. However, we shall return to it later, but focusing our attention on the case of linear systems of plane curves.

3.2. Cones of curves of rational surfaces and P-sufficient configurations. As we shall see along this paper, techniques related to the cone of curves of a projective regular rational surface have fruitful applications in several problems in Algebraic Geometry. So, we include here a brief exposition of the basic properties of the cone of curves, and also we recall the notion, introduced in [27] and [28], of P-sufficient configuration (over a relatively minimal rational surface). This concept depends only on the P-Enriques diagram of the configuration and it implies the polyhedrality of the cone of curves associated with the surface obtained by blowing-up 
the points of the configuration. The obtention of conditions implying the polyhedrality of the cone of curves is an interesting issue, as we shall see in the applications. Let $Z$ be a regular projective rational surface and consider its cone of curves $N E(Z) \subseteq A_{1}(Z)$ and its closure with respect to the real topology, denoted by $\overline{N E}(Z)$. Notice that, in this case, we can identify the spaces $A_{1}(Z)$ and $A^{1}(Z)$; we shall denote them by $A(Z)$. We shall assume that $\operatorname{dim} A(Z) \geq 3$ (otherwise the cone of curves is regular). Recall that, if $C$ is a convex cone of $A(Z)$, a face of $C$ is a sub-cone $F \subseteq C$ such that $a+b \in F$ implies that $a, b \in F$, for all pair of elements $a, b \in C$. The 1-dimensional faces of $C$ are the extremal rays of $C$. Fix an ample divisor $H$ on $Z$. By Kleiman's ampleness criterion [43], $H \cdot x>0$ for all $x \in \overline{N E}(Z) \backslash\{0\}$ and, hence, the cone $\overline{N E}(Z)$ is strongly convex. This implies that it is generated by its extremal rays. Consider the cone

$$
Q(Z)=\left\{x \in A(Z) \mid x^{2} \geq 0, H \cdot x \geq 0\right\} .
$$

By the Hodge index theorem [39, V.1.9] there exists a basis of $A(Z)$ for which the intersection bilinear form on $A(Z)$ is given by the diagonal matrix $\operatorname{diag}(1,-1, \ldots,-1)$ in such a way that $Q(Z)$ is defined by an inequality of the type $x_{1} \geq\left(\sum_{i=2}^{\rho(Z)} x_{i}^{2}\right)^{1 / 2}$ in the suitable coordinates. Then, $Q(Z)$ is the half-cone over an Euclidean ball of dimension $\rho(Z)-1$, which is strictly convex. One has that $Q(Z) \subseteq \overline{N E}(Z)$ [44, II.4.12.1] and, therefore, the extremal rays of $\overline{N E}(Z)$ must be spanned by elements $x \in A(Z)$ such that $x^{2} \leq 0$. The extremal rays of $\overline{N E}(Z)$ which are not in $Q(Z)$ are spanned by classes of integral curves $C$ with $C^{2}<0$ [44, II.4.12.3]. Moreover, if $C$ is an integral curve on $Z$ such that $C^{2}<0$ then $C$ generates an extremal ray of $\overline{N E}(Z)[44$, II.4.12.2]. The extremal rays of $N E(Z)$ generated by elements $x$ such that $K_{Z} \cdot x<0$ ( $K_{Z}$ being a canonical divisor on $Z$ ) are known as a consequence of the Mori cone theorem (see [44, III.1] for instance): they are exactly those spanned by the images in $A(Z)$ of the $(-1)$-curves (that is, integral regular rational curves whose self-intersection is equal to -1$)$; furthermore, if there are infinitely many $(-1)$-curves, the accumulation points of the set of generated extremal rays must be on the orthogonal hyperplane to the canonical class, $K_{Z}^{\perp}$. However, very little is known concerning the region $N E(Z) \cap\left(K_{Z} \cdot x \geq 0\right)$. From the classical theory of surfaces, it is well-known that $Z$ can be obtained by blowing-up the points of a configuration $\mathcal{C}$ over a relatively minimal rational surface $X$, that can be either the projective plane $\mathbb{P}^{2}$ of a Hirzebruch surface $\mathbb{F}_{a}:=$ $\mathbb{P}\left(\mathcal{O}_{\mathbb{P}^{1}} \oplus \mathcal{O}_{\mathbb{P}^{1}}(a)\right), a$ being a non negative integer, $a \neq 1$. We fix, from now on, both $\mathcal{C}$ and $X$ such that $Z=X_{\mathcal{C}}$. When $K_{Z}^{2} \geq 0$ we have the following results:

São Paulo J.Math.Sci. 3, 1 (2009), 115-160 
(i) If $K_{Z}^{2}>0$ then $N E(Z)$ is polyhedral (see [53] and [27, Cor. 1(i)] for the case in which $X$ is the projective plane, and [28, Th. 2(a)] for the general case). Notice that this happens if and only if the cardinality of $\mathcal{C}$ is $\leq 8$ (resp. 7 ), whenever $X=\mathbb{P}^{2}$ (resp. $X$ is a Hirzebruch surface).

(ii) If $K_{Z}^{2}=0$, then either $N E(Z)$ is polyhedral, or the set of extremal rays of $\overline{N E}(Z)$ has a unique accumulation point, which is spanned by $-K_{Z}$ (see [27, Cor. 1(ii)] and [28, Th. 2(b)]). Notice that $K_{Z}^{2}=0$ if and only if the cardinality of $\mathcal{C}$ is 9 (resp. 8), whenever $X=\mathbb{P}^{2}$ (resp. $X$ is a Hirzebruch surface).

(iii) If $K_{Z}^{2}=0$ and $K_{Z} \cdot D>0$ for some effective divisor $D$ on $Z$, then $N E(Z)$ is polyhedral [28, Th. 2(c)].

The cone of curves is not, in general, polyhedral. For instance, in $[8$, Example 4.3] it is provided an example of a chain constellation of 9 points over $X=\mathbb{P}^{2}$ such that the surface $X_{\mathcal{C}}$ has infinitely many $(-1)$-curves and, therefore, it has infinitely many extremal rays. The following result provides conditions for the polyhedrality and regularity of $N E(Z)$ and the characteristic cone $\tilde{P}(X)$ in terms of the existence of curves passing through a certain cluster.

Proposition 3.1. [28, Sect. 2.2.2] Set $\mathcal{C}=\left\{Q_{0}, \ldots, Q_{n}\right\}$ and consider the cluster $\mathcal{K}=(\mathcal{C}, \underline{m})$, where $m_{i}=1,0 \leq i \leq n$. Assuming that $X=\mathbb{P}^{2}$ the following properties hold:

(a) If there exists a line passing through $\mathcal{K}$ then the cones $N E(Z)$ and $P(Z)$ are regular, $N E(Z)$ being generated by $E_{0}, \ldots, E_{n}$ and the image in $A(Z)$ of the strict transform of the line.

(b) If there exists a conic $C$ passing through $\mathcal{K}$, then $N E(Z)$ is a polyhedral cone generated by $E_{0}, \ldots, E_{n}$, the images in $A(Z)$ of the strict transforms of the lines passing through two points in $\mathcal{C}$, and the image of the virtual transform of $C$ in $Z$ with respect to $\mathcal{K}$. Moreover $\tilde{P}(Z)$ is a closed cone.

(c) If there exists a conic passing through $\mathcal{K}$ and $n \leq 3$ then $N E(Z)$ and $\tilde{P}(Z)$ are regular cones.

(d) if $n \geq 4$ and there exists an integral conic such that its successive strict transforms pass through $Q_{0}, \ldots, Q_{4}$, then $\tilde{P}(Z)$ is not simplicial.

Assuming that $X$ is a Hirzebruch surface $\mathbb{F}_{a}, N E(Z)$ is polyhedral whenever a curve in the linear system $|(1-a) F+2 M|$ pass through $\mathcal{K}, F$ being a fiber of the natural morphism $\mathbb{F}_{a} \rightarrow \mathbb{P}^{1}$ and $M$ being the divisor of zeros of a non-trivial global section of $\mathcal{O}_{\mathbb{F}_{a}}(1)$. 
Now, we define the above mentioned notion of P-sufficient configuration.

Definition 3.3. Set $\mathcal{C}=\left\{Q_{0}, \ldots, Q_{n}\right\}$ and consider the divisors $D\left(Q_{i}\right)$ such that $\mathcal{P}_{Q_{i}} \mathcal{O}_{Z}=\mathcal{O}_{Z}\left(-D\left(Q_{i}\right)\right), \mathcal{P}_{Q_{i}}$ being the simple complete ideal of $\mathcal{O}_{X, O}$ associated with $Q_{i}, O$ being the image of $Q_{i}$ on $X$ (see Section 2.2). Consider the $(n+1)$-dimensional symmetric matrix $G:=\left(\left(g_{i j}\right)\right)$, where

$$
g_{i j}=-\alpha D\left(Q_{i}\right) \cdot D\left(Q_{j}\right)-\left(K_{X_{\mathcal{C}}} \cdot D\left(Q_{i}\right)\right)\left(K_{X_{\mathcal{C}}} \cdot D\left(Q_{j}\right)\right),
$$

$\alpha$ being 9 (resp. 8) if $X=\mathbb{P}^{2}$ (resp. $X$ is a Hirzebruch surface). The configuration $\mathcal{C}$ is said to be $P$-sufficient if $x G x^{t}>0$ for all vectors $\left(x_{0}, \ldots, x_{n}\right) \in$ $\mathbb{R}^{n+1} \backslash\{0\}$ such that $x_{i} \geq 0$ for all $i$.

Remark 3.1. Recall that the coefficients of each divisor $D\left(Q_{i}\right)$ are those appearing in the $i$ th row of the inverse of the proximity matrix of $\mathcal{C}$. Hence, the matrix $G$ is easy to compute and depends only on the proximity relations among the points in $\mathcal{C}$.

A general method to decide if a configuration is $\mathrm{P}$-sufficient or not is given in [26], which consists of checking the non-emptiness of certain sets defined by linear inequalities. Also, a configuration is $\mathrm{P}$-sufficient whenever all the entries of the matrix $G$ are non-negative and the diagonal ones are strictly positive (for an example, see [27, page 86]). Furthermore, when the configuration is a chain, it is very easy to decide if it is P-sufficient or not:

Proposition 3.2. [28, Cor. 2] When $\mathcal{C}$ is a chain constellation, $\mathcal{C}$ is $P$ sufficient if and only if the last entry of the matrix $G$ is strictly positive.

The following result is proved in [27, Th. 2] when $X=\mathbb{P}^{2}$ and in [28, Th. 1] in the general case, and it shows that the P-sufficient configurations give raise to surfaces with polyhedral cones of curves.

Theorem 3.1. If $\mathcal{C}$ is a P-sufficient configuration then the cone of curves $N E(Z)$ is polyhedral.

Remark 3.2. It can be proved that, if $X=\mathbb{P}^{2}$ (resp. $X$ is a Hirzebruch surface) and the cardinality of $\mathcal{C}$ is $\leq 8$ (resp. $\leq 7$ ) then $\mathcal{C}$ is P-sufficient. For an example of a $\mathrm{P}$-sufficient configuration with 11 points see $[27$, page 86].

Remark 3.3. If $N E(Z)$ is polyhedral, the configuration $\mathcal{C}$ may not be $\mathrm{P}$ sufficient. For example, take a configuration consisting of 9 or more proper points on a conic. The cone $N E(Z)$ is polyhedral (by Proposition 3.1) but, however, the configuration is not P-sufficient.

Remark 3.4. As a result which follows from [43], the topological cells of the characteristic cone $\tilde{P}(Z)$ (see [43, page 340] for the definition) correspond one to one to surjective morphisms from $Z$ to a (connected) normal 
variety (contractions). Since there is an injection between the set of topological cells of $\tilde{P}(Z)$ and the one of $P(Z)$ [8, Th. 2.1], one has that the polyhedrality of $N E(Z)$ implies that the number of contractions is finite.

3.3. Clusters of base points associated with pencils on surfaces. We shall consider now a particular type of linear systems on a projective regular surface $X$ : given an effective divisor $H, \varrho$ will be a linear subsystem of $|H|$ without fixed components and with projective dimension 1 (a pencil in the sequel). Such a pencil $\varrho$ corresponds to the projectivization of the sub-vector space $V_{\varrho}$ of $H^{0}\left(X, \mathcal{O}_{X}(H)\right)$ given by $\left\{s \in H^{0}\left(X, \mathcal{O}_{X}(H)\right) \mid\right.$ $\left.(s)_{0} \in \varrho\right\} \cup\{0\},(s)_{0}$ denoting the divisor of zeros of the section $s$. If $\mathcal{I}(\varrho)$ is the ideal sheaf on $X$ defining the base point scheme of $\varrho$, consider the associated cluster of base points $\mathcal{K}_{\mathcal{I}(\varrho)}=\left(\mathcal{C}_{\mathcal{I}(\varrho)}, \underline{m}\right)$ and the associated divisor $D\left(\mathcal{K}_{\mathcal{I}(\varrho)}\right)$.

\section{Cones of curves and irreducible pencils}

One can consider the linear system on $X$ of all effective divisors in $|H|$ passing through the cluster $\mathcal{K}_{\mathcal{I}(\varrho)}$, which will be denoted by $\mathcal{L}_{H}\left(\mathcal{K}_{\mathcal{I}(\varrho)}\right)$. Then, it is clear that $\varrho \subseteq \mathcal{L}_{H}\left(\mathcal{K}_{\mathcal{I}(\varrho)}\right)$. The question we propose to answer now is the following one: when is this inclusion an equality? or, equivalently, when is a pencil determined by the class of $H$ in the Picard group and its cluster of base points? A fixed basis of $V_{\varrho}$ provides a rational map $f: X \cdots \rightarrow \mathbb{P} V_{\varrho} \cong \mathbb{P}^{1}$ (actually this map is independent from the basis up to composition with an automorphism of $\mathbb{P}^{1}$ ). The closures of the fibers of $f$ are exactly the curves of the pencil $\varrho$. For this reason, the elements of $\varrho$ are usually called fibers. Moreover, the morphism $\sigma:=\sigma_{\mathcal{C}_{\mathcal{I}(\varrho)}}: Z:=X_{\mathcal{C}_{\mathcal{I}(\varrho)}} \rightarrow X$ is defined by the virtual transform on $Z$ of the chosen basis of $V_{\varrho}$ with respect to the cluster of base points $\mathcal{K}_{\mathcal{I}(\varrho)}$, and it is a minimal composition of point blowing-ups eliminating the indeterminacies of the rational map $f$, that is, the map $h:=f \circ \sigma: Z \rightarrow \mathbb{P}^{1}$ is a morphism (see [4, Th. II.7]). It is clear that, if $C_{1}$ and $C_{2}$ are two curves on $Z$ such that $C_{1}+C_{2}$ is contracted (to a closed point) by $h$, then both curves $C_{1}, C_{2}$ must also be contracted by $h$. Therefore, the images in $A(Z)$ of all curves of $Z$ which are contracted by $h$ generate a face of the cone of curves $N E(Z)$, that we shall denote by $\Delta_{\varrho}$. Consider the divisor $G_{\varrho}:=\sigma^{*} H-D\left(\mathcal{K}_{\mathcal{I}(\varrho)}\right)$. The linear system $\varrho$ on $Z$ given by the virtual transforms of the curves in $\varrho$ with respect to the cluster of base points is contained in the complete linear system $\left|G_{\varrho}\right|$, and the image of $G_{\varrho}$ in $A(Z)$ is the same than the one of a general fiber of the pencil $\varrho$. As a consequence, $G_{\varrho}$ is a nef divisor (since its associated complete linear system is base point free). Therefore, we conclude that $\Theta_{\varrho}:=N E(Z) \cap G_{\varrho}^{\perp}$ is a face of $N E(Z)$. 
Lemma 3.1. Both faces $\Delta_{\varrho}$ and $\Theta_{\varrho}$ coincide.

Proof. The morphism $h$ factorizes as $h=t \circ \phi$, where

$\phi: Z \rightarrow \mathbb{P} H^{0}\left(Z, \mathcal{O}_{Z}\left(G_{\varrho}\right)\right)$ is the morphism induced by a basis of

$H^{0}\left(Z, \mathcal{O}_{Z}\left(G_{\varrho}\right)\right)$ obtained by completing the one given by the virtual transform of the fixed basis of $V_{\varrho}$, and $t: \mathbb{P} H^{0}\left(Z, \mathcal{O}_{Z}\left(G_{\varrho}\right)\right)--\rightarrow \mathbb{P} V_{\varrho} \cong \mathbb{P}^{1}$ is the projection. A curve $C$ is contracted by $\phi$ if and only if $G_{\varrho} \cdot C=0$, and it is obvious that, in this case, it is also contracted by $h$. Therefore, one has that $\Theta_{\varrho} \subseteq \Delta_{\varrho}$. Since the strict transforms on $Z$ of two general fibers do not meet, one has that $G_{\varrho}^{2}=0$. When $\operatorname{dim} A(Z) \geq 3$, the hyperplane $G_{\varrho}^{\perp}$ is tangent to the cone $Q(Z)$ defined in the preceding section. So, we have the following equivalence (which is also valid when $\operatorname{dim} A(Z)=2$ ):

$x \in G_{\varrho}^{\perp} \backslash\{0\}$ and $x^{2}<0$ if and only if $x$ is not a (real) multiple of $G_{\varrho}$.

It is clear that there exists $y \in P(Z) \backslash\{0\}$ such that $\Delta_{\varrho} \subseteq N E(Z) \cap y^{\perp}$. But $y$ belongs to $G_{\varrho}^{\perp}$, since $G_{\varrho} \in \Delta_{\varrho}$. So, by the above equivalence, $y$ is a multiple of $G_{\varrho}$ and hence $\Delta_{\varrho} \subseteq \Theta_{\varrho}$.

Notice that the integral curves which are contracted by $h$ are exactly the strict transforms of the integral components of the fibers of the pencil $\varrho$ and some strict transforms of exceptional divisors (the so-called vertical exceptional divisors). From this consideration and the above ones it follows the next result, whose proof is also implicit in [29] but in a different framework.

Proposition 3.3. An integral curve $C$ on $X$ is a component of a fiber of the pencil $\varrho$ if and only if its strict transform $\tilde{C}$ on $Z$ satisfies that $G_{\varrho} \cdot \tilde{C}=0$. Moreover, in this case, $\tilde{C}^{2} \leq 0$.

We fix a closed immersion $i: X \hookrightarrow \mathbb{P}^{s}$ of $X$ into a projective space; the degree of a curve $F$ on $X$ will be the intersection product $i^{*} \mathcal{O}_{\mathbb{P}^{s}}(1) \cdot F$.

The pencil $\varrho$ is said to be irreducible if it has integral general fibers. The following proposition is also proved in [29] when $X$ is the projective plane using the Cayley-Bacharach Theorem, but we show here a different proof.

Proposition 3.4. If $\varrho$ is irreducible then $\varrho=\mathcal{L}_{H}\left(\mathcal{K}_{\mathcal{I}(\varrho)}\right)$, that is, it is determined by the class of $H$ in the Picard group and its cluster of base points.

Proof. Reasoning by contradiction, assume that the projective dimension of $\mathcal{L}_{H}\left(\mathcal{K}_{\mathcal{I}(\varrho)}\right)$ is greater than 1 . Take a curve $C \in \mathcal{L}_{H}\left(\mathcal{K}_{\mathcal{I}(\varrho)}\right)$ such that $C \notin \varrho$. The image in $A(Z)$ of the virtual transform of $C$ on $Z$ with respect to the cluster $\mathcal{K}_{\mathcal{I}(\varrho)}$ belongs to $\left|G_{\varrho}\right|$ and therefore, if $\tilde{C}$ denotes the strict 
transform, it holds that $G_{\varrho} \cdot \tilde{C}=0$ (taking into account that $G_{\varrho}$ is nef and $G_{\varrho}^{2}=0$ ). Then, applying the above result, one has that the integral components of $C$ are fibers of the pencil $\varrho$. If $C_{1}$ denotes one of these components, one has that its degree is strictly less than the one of the general fibers of $\varrho$ (otherwise, on the one hand, $C_{1}$ would be a fiber of the pencil and, on the other hand, it would coincide with $C$, a contradiction). But, since the pencil is irreducible, the number of reducible fibers is finite. The contradiction follows from the fact that, by the initial assumption, there are infinitely many curves $C$ as above.

Assuming that the characteristic of the ground field is 0 we prove the converse of the above statement:

Proposition 3.5. If char $(\mathbb{K})=0$ then $\varrho$ is an irreducible pencil if and only if $\varrho=\mathcal{L}_{H}\left(\mathcal{K}_{\mathcal{I}(\varrho)}\right)$.

Proof. Reasoning by contradiction, assume that $\varrho=\mathcal{L}_{d}\left(\mathcal{K}_{\mathcal{I}(\varrho)}\right)$ and $\varrho$ is not irreducible. Then $\varrho$ is composite with an irreducible pencil, that is, there exist rational maps $q_{1}: X \cdots \rightarrow \mathbb{P}^{1}$ and $q_{2}: \mathbb{P}^{1} \cdots \rightarrow \mathbb{P}^{1}$ such that the closures of the fibers of $q_{1}$ correspond to an irreducible pencil $\varsigma$ of degree $e$, $q_{2}$ is generically finite of degree $n:=d / e>1$ and $f=q_{2} \circ q_{1}$. Therefore, if $C$ is a general fiber of $\varsigma$, taking into account that $n \tilde{C}$ is linearly equivalent to $G_{\varrho}$, one gets that $n C$ is a fiber of $\varrho$. Hence, general fibers of $\varrho$ are not reduced, which is a contradiction.

\section{Pencils at infinity}

We shall assume until the end of this subsection that $\operatorname{char}(\mathbb{K})=0$. A specially interesting class of pencils on the projective plane $\mathbb{P}^{2}$ are the socalled pencils at infinity.

Definition 3.4. Taking homogeneous coordinates $\left(X_{1}: X_{2}: X_{3}\right)$ on $\mathbb{P}^{2}$, a (linear) pencil (without fixed components) $\varrho \subseteq\left|\mathcal{O}_{\mathbb{P}^{2}}(d)\right|, d \in \mathbb{Z}_{+}$, is said to be at infinity if $V_{\varrho}=\left\langle F, X_{3}^{d}\right\rangle$, where $F\left(X_{1}, X_{2}, X_{3}\right)$ is an homogeneous polynomial of degree $d$ and $X_{3}=0$ is considered as the line of infinity.

A particular case of pencil at infinity is obtained when $F=0$ defines a curve $C$ having one place at infinity, that is, it intersects with the line of infinity only in a single point $Q$ and $C$ is reduced and unibranched at $Q$. This is easily seen to imply that $C$ is integral. This type of curves have been extensively studied by several authors as Abhyankar, Moh, Satayhe and Suzuki $[1,2,3,57,64,67]$. All the curves in the pencil at infinity defined by $F$, except the non-reduced one, have one place at infinity and their singularities at the point of infinity have the same minimal embedded 
resolution than the one of $C$ [57]. In [10] is proved a structure theorem for the cone of curves and the characteristic cone of the surface $Z$ obtained by blowing-up the configuration of base points of a pencil of this type (which is a chain constellation). Actually, instead of the cone of curves, the effective semigroup is considered; it is the sub-semigroup $N E_{S}(Z)$ of $\operatorname{Pic}(Z)$ spanned by the classes of the effective divisors.

Theorem 3.2. [10] Let $\varrho$ be a pencil at infinity such that $V_{\varrho}=\left\langle F, X_{3}^{d}\right\rangle$, where $F=0$ defines a curve having one place at infinity of degree $d \geq 1$, and let $Z$ be the surface obtained by blowing-up the points in the configuration $\mathcal{C}_{\mathcal{I}(\varrho)}$. Then:

(a) The semigroup $N E_{S}(Z)$ is spanned by the strict transform of the line of infinity and the strict transforms of the exceptional divisors.

(b) The cones $P(Z)$ and $\tilde{P}(Z)$ coincide and are regular.

Recall that, in the local case, Enriques solved the problem of determining when there exists a germ of curve passing effectively through a cluster $(\mathcal{C}, \underline{m})$ leading to the proximity inequalities [22, 13], and Zariski considered the semigroup of $\sigma_{\mathcal{C}}$-generated line bundles in order to establish the unique factorization of complete ideals [71]. In our case, global analogues to these problems consist of characterizing the semigroup $P_{S}^{s t}(Z) \subseteq \operatorname{Pic}(Z)$ generated by the classes of the strict transforms on $Z$ of curves on $\mathbb{P}^{2}$, and the semigroup $\tilde{P}_{S}(Z) \subseteq \operatorname{Pic}(Z)$ generated by the classes of divisors $D$ on $Z$ such that $\mathcal{O}_{\mathbb{P}^{2}}(D)$ is generated by global sections (notice that one has $\tilde{P}_{S}(Z) \subseteq P_{S}^{s t}(Z) \subseteq P_{S}(Z)$, where $P_{S}(Z)$ denotes the semigroup generated by the nef classes). Concerning these questions, in [10] it is proved the following result:

Theorem 3.3. Let $\varrho$ and $Z$ be as in Theorem 3.2.

(a) A divisor class $D$ belongs to $P_{S}^{s t}(Z)$ if and only if $\mathcal{O}_{\mathbb{P}^{2}}(D)$ is generated by global sections except possibly at finitely many closed points.

(b) $\tilde{P}_{S}(Z)=P_{S}^{s t}(Z)=P_{S}(Z)$ if and only if all curves in the pencil $\varrho$ (except the non-reduced one) are rational.

Due to Theorem 3.2 the cone of curves $N E(Z)$ associated to a pencil defined by a curve having one place at infinity is always polyhedral. Since $\mathrm{P}$-sufficient configurations give rise to polyhedral cones of curves, a natural question arises: when is the configuration of base points of such a pencil P-sufficient? The answer is given in the following proposition:

Proposition 3.6. [54, Prop. 3] Let $\varrho$ and $Z$ be as in Theorem 3.2. The configuration $\mathcal{C}_{\mathcal{I}(\varrho)}$ is $P$-sufficient if and only if $F=0$ defines an AbhyankarMoh-Suzuki curve (i.e. it is rational and smooth in its affine part). 
Consider now a pencil at infinity $\varrho$ given by an homogeneous polynomial $F$ of degree $d \geq 1$ but not necessarily defining a curve having one place at infinity. Assuming certain conditions on $F$ it is possible to describe the structure of the effective semigroup and the characteristic cone of the surface $Z$ obtained by blowing-up the configuration of base points of the pencil:

Theorem 3.4. [11, Th. 3] Assume that $\varrho$ is an irreducible pencil at infinity such that $F$ factorizes as $F_{1}^{a_{1}} \cdots F_{s}^{a_{s}}$, where $d_{1}, \ldots, d_{s}, a_{1}, \ldots, a_{s} \in \mathbb{Z}_{+}$, $\operatorname{gcd}\left(a_{1}, \ldots, a_{s}\right)=1, F_{1}, \ldots, F_{s}$ are homogeneous polynomials of respective degrees $d_{1}, \ldots, d_{s}$ such that the curves defined by $F_{i}=0$ have one place at infinity and, if $s \geq 2, F_{i} \notin\left\langle F_{1}, Z^{d_{1}}\right\rangle$ for some $i, 2 \leq i \leq s$. Then,

(a) The effective semigroup $N E_{S}(Z)$ is spanned by the strict transforms on $Z$ of the following curves: the exceptional divisors, the line of infinity and the curves defined by the polynomials $F_{i}, 1 \leq i \leq s$.

(b) $\tilde{P}(Z)=P(Z)$.

\section{Applications}

4.1. Applications to the Poincaré Problem. Some progress concerning the theory of foliations have been done by using, as a tool, the language of configurations and clusters and, also, considerations involving cones of curves (sections 3.2 and 3.3). They are related to the so-called Poincaré problem. We shall summarize some of such progress but, previously, we will introduce briefly some background on the theory of foliations.

\section{Background}

We shall assume that $\mathbb{K}=\mathbb{C}$. An (algebraic singular) foliation $\mathcal{F}$ on a projective smooth surface (a surface in the sequel) $X$ can be defined by a collection $\left\{\left(U_{i}, \omega_{i}\right)\right\}_{i \in I}$, where $\left\{U_{i}\right\}_{i \in I}$ is an open covering of $X, \omega_{i}$ is a non-zero regular differential 1-form on $U_{i}$ with isolated zeros and, for each couple $(i, j) \in I \times I$,

$$
\omega_{i}=g_{i j} w_{j} \quad \text { on } \quad U_{i} \cap U_{j}, \quad g_{i j} \in \mathcal{O}_{X}\left(U_{i} \cap U_{j}\right)^{*} .
$$

The singular locus $\operatorname{Sing}(\mathcal{F})$ of $\mathcal{F}$ is the discrete subset of $X$ defined by

$$
\operatorname{Sing}(\mathcal{F}) \cap U_{i}=\text { zeroes of } w_{i} .
$$

The transition functions $g_{i j}$ of a foliation $\mathcal{F}$ define an invertible sheaf $\mathcal{L}$ on $X$ and the relations (2) can be thought as defining relations of a global section of the sheaf $\mathcal{L} \otimes \Omega_{X}^{1}$, which has isolated zeros (because each $\omega_{i}$ has isolated zeros). This section is uniquely determined by the foliation $\mathcal{F}$, up to multiplication by a non zero element in $\mathbb{C}$. Conversely, given an invertible sheaf $\mathcal{L}$ on $X$, any global section of $\mathcal{L} \otimes \Omega_{X}^{1}$ with isolated zeros defines a 
foliation $\mathcal{F}$. Alternatively, a foliation can also be defined by a collection $\left\{\left(U_{i}, v_{i}\right)\right\}_{i \in I}$, where $v_{i}$ is a vector field on $U_{i}$ with isolated zeroes, satisfying analogous relations as in (2). Given $P \in X$, a (formal) solution of $\mathcal{F}$ at $P$ will be an irreducible element $f \in \widehat{\mathcal{O}}_{X, P}$ (where $\widehat{\mathcal{O}}_{X, P}$ is the $\mathrm{m}_{P}$-adic completion of the local ring $\mathcal{O}_{X, P}$ and $\mathrm{m}_{P}$ its maximal ideal) such that the local differential 2-form $\omega_{P} \wedge d f$ is a multiple of $f, w_{P}$ being a local equation of $\mathcal{F}$ at $P$. An element in $\widehat{\mathcal{O}}_{X, P}$ will be said to be invariant by $\mathcal{F}$ if all its irreducible components are solutions of $\mathcal{F}$ at $P$. An algebraic solution of $\mathcal{F}$ will be an integral (i.e. reduced and irreducible) curve $C$ on $X$ such that its local equation at each point in its support is invariant by $\mathcal{F}$. Moreover, if every integral component of a curve $D$ on $X$ is an algebraic solution, we shall say that $D$ is invariant by $\mathcal{F}$. Seidenberg's result of reduction of singularities [66] proves that there is a sequence of blowing-ups

$$
X_{n+1} \stackrel{\pi_{n}}{\longrightarrow} X_{n} \stackrel{\pi_{n-1}}{\longrightarrow} \cdots \stackrel{\pi_{2}}{\longrightarrow} X_{2} \stackrel{\pi_{1}}{\longrightarrow} X_{1}:=X
$$

such that the strict transform $\mathcal{F}_{n+1}$ of $\mathcal{F}$ on the last obtained surface $X_{n+1}$ has only certain type of singularities which cannot be removed by blowingup, called simple singularities. Such a sequence of blowing-ups is called a resolution of $\mathcal{F}$, and it will be minimal if it is so with respect to the number of involved blowing-ups. Assuming that the above sequence of blowingups is a minimal resolution of $\mathcal{F}$, we shall denote by $\mathcal{C}_{\mathcal{F}}$ the associated configuration $\left\{P_{i}\right\}_{i=1}^{n}$ given by the centers of the blowing-ups. Note that each point $P_{i}$ is an ordinary (that is, not simple) singularity of the foliation $\mathcal{F}_{i}$. An exceptional divisor $B_{P_{i}}$ (respectively, a point $P_{i} \in \mathcal{C}_{\mathcal{F}}$ ) is called non-dicritical if it is invariant by the foliation $\mathcal{F}_{i+1}$ (respectively, all the exceptional divisors $B_{P_{j}}$, with $P_{j} \geq P_{i}$, are non-dicritical). Otherwise, $B_{P_{i}}$ (respectively, $P_{i}$ ) is said to be dicritical. Particularizing to the projective plane, it holds that a foliation $\mathcal{F}$ on $\mathbb{P}^{2}$ (of degree $r$ ) can be defined by means of a projective 1 -form

$$
\Omega=A d X_{1}+B d X_{2}+C d X_{3},
$$

where $A, B$ and $C$ are homogeneous polynomials of degree $r+1$ without common factors which satisfy the Euler's condition $X_{1} A+X_{2} B+X_{3} C=0$ (see [33]). From a more geometrical point of view, $\mathcal{F}$ can be regarded as the rational map $\Phi: \mathbb{P}^{2} \cdots \rightarrow \check{\mathbb{P}}^{2}$ which sends a point $P$ to $(A(P): B(P)$ : $C(P)$ ). The singular locus of $\mathcal{F}$ is the set of points where this rational map is not defined, that is, the set of common zeros of the polynomials $A, B$ and $C$. Moreover, a curve $D$ on $\mathbb{P}^{2}$ is invariant by $\mathcal{F}$ if, and only if, $G$ divides the projective 2 -form $d G \wedge \Omega$, where $G\left(X_{1}, X_{2}, X_{3}\right)=0$ is an equation of $D$. 


\section{The Poincaré problem}

We begin with a differential equation with polynomial coefficients of order 1 and degree 1 , that is, of the type $Q(x, y) y^{\prime}+P(x, y)=0$, with $P, Q \in \mathbb{C}[x, y]$ or, in a more general form, given by the vector field $D=$ $Q(x, y) \partial / \partial x-P(x, y) \partial / \partial y$ or, equivalently, the differential form $\omega=$ $P(x, y) d x+Q(x, y) d y$. It is said that the differential equation is algebraically integrable or that it has a rational first integral if there exists a rational function $R=\frac{f}{g}, f, g \in \mathbb{C}[x, y]$, such that $\omega \wedge d R=0$ (or equivalently $D(R)=0$ ). This implies that the function $R$ is constant on the solutions of the equation, that is, these are the curves whose implicit equations are of the form $\lambda f+\mu g=0, \lambda, \mu \in \mathbb{C}$ (hence all solutions are algebraic curves). In 1891, H. Poincaré [60] observed that, once we possess a bound on the degree of a polynomial defining a general irreducible solution, we can try to find the rational first integral by making purely algebraic computations. The problem of finding this bound in terms of the degree of the foliation is classically known as the Poincaré problem, although it was studied before by Darboux and also by Painlevé and Autonne more or less at the same time than Poincaré. From a more modern point of view, a vector field on the affine plane is given by polynomial coefficients if and only if it is the restriction of a foliation of the projective plane. So, the Poincaré problem can be treated in this framework. Then, we shall say that a foliation $\mathcal{F}$ of $\mathbb{P}^{2}$ has a rational first integral if there exists a rational function $R$ of $\mathbb{P}^{2}$ such that $d R \wedge \Omega=0$. The substantial current interest in the Poincaré problem was stimulated by Cerveau and Lins Neto in [15]. In this paper, the problem is stated in a more general form, avoiding the assumption of the algebraic integrability. That is, if we assume that a foliation $\mathcal{F}$ of $\mathbb{P}^{2}$ has an algebraic solution $C$, can we give conditions that allow us to bound $\operatorname{deg} C$ in terms of $\operatorname{deg} \mathcal{F}$ ? The main result of [15] gives an answer assuming that all the singularities of $C$ are simple nodes (in this case $\operatorname{deg} C \leq \operatorname{deg} \mathcal{F}+2$ ). Carnicer, in [12], proves the same inequality in the case that $C$ does not pass through dicritical singularities of $\mathcal{F}$. However, there exist examples showing that, in general, $\operatorname{deg} C$ cannot be bounded in terms of $\operatorname{deg} \mathcal{F}$. A remarkable counterexample is given in [47] (families of algebraically integrable foliations of fixed degree and singularities of fixed analytic type are given, in such a way that the general algebraic solutions have arbitrarily big degree).

Results using infinitely near points

In [5], Carnicer and the first author extend in certain manner the result given in [12] when dicritical singularities appear. They use, as an important tool and unifying element in the paper, the language of infinitely near points and proximity. In fact, they prove proximity formulae for foliations [5, Prop. 
3.5] and use them to give relations between local invariants of an algebraic solution and local invariants of the foliation. To state the main result, we need to introduce some notations.

Given a reduced invariant curve $C$ of a foliation $\mathcal{F}$ of a projective smooth surface $X$, let $\mathcal{N}_{C}$ be the configuration over $X$ that consists of those points in $\mathcal{C}_{\mathcal{F}}$ whose image on $X$ by the composition of blowing-ups given in (3) belongs to $C$. For each $P \in \mathcal{N}_{C}$ denote by $s_{P}(\mathcal{F})$ the number of points $Q \in \mathcal{N}_{C}$ such that $P \rightarrow Q$ and the exceptional divisor $B_{Q}$ is non-dicritical, and set $\nu_{P}(C)\left(\right.$ resp. $\left.\nu_{P}(\mathcal{F})\right)$ the multiplicity at $P$ of the strict transform of $C$ (resp. the minimum order of the coefficients of a local differential form defining the strict transform of $\mathcal{F}$ at $P$ ).

Theorem 4.1. [5, Th. 1] Let $\mathcal{F}$ be a foliation of $\mathbb{P}^{2}$ and $C$ a reduced curve which is invariant by $\mathcal{F}$. Let $\mathcal{K}=\left(\mathcal{N}_{C}=\left\{Q_{i}\right\}_{i=1}^{t}, \underline{m}\right)$ be the cluster such that $m_{i}:=\nu_{Q_{i}}(C)+s_{Q_{i}}(\mathcal{F})-\nu_{Q_{i}}(\mathcal{F})-1$ for all $i \in\{1, \ldots, t\}$. Let $d$ be a non-negative integer such that the linear system $\mathcal{L}_{d}(\mathcal{K})$ (of curves of degree $d$ passing through $\mathcal{K})$ is not empty. Then:

$$
\operatorname{deg}(C) \leq \operatorname{deg}(\mathcal{F})+2+d .
$$

As an application, if either we fix the number of tangents at the singular points of $\mathcal{F}$, or we fix the equisingularity types of the curve at the singular points of $\mathcal{F}$, then concrete values of $d$ can be obtained from the fixed data computing the linear system $\mathcal{L}_{d}(\mathcal{K})$ (this involves the resolution of a system of linear equations). The obtained bounds will be valid for particular types of invariant curves. It is worth adding that the above mentioned results of Cerveau and Lins Neto, and Carnicer are particular cases of Theorem 4.1 since, in both cases, it can be proved that $\nu_{Q_{i}}(\mathcal{F})+1 \geq \nu_{Q_{i}}(C)+$ $s_{Q_{i}}(\mathcal{F})$ for all $Q_{i} \in \mathcal{N}_{C}$ (then, the results follow by taking $d=0$ ). In [7], the result given in Theorem 4.1 is generalized for foliations of arbitrary projective smooth surfaces. In this case, one looses the concept of degree and, in addition to configurations and proximity, the use of divisors and Intersection Theory is required. The main result is the following one:

Theorem 4.2. [7, Th. 2] Let $X$ be a projective smooth algebraic surface, $\mathcal{F}$ a foliation of $X, C$ a reduced curve which is invariant by $\mathcal{F}$ and $\mathcal{K}=$ $\left(\mathcal{N}_{C}, \underline{m}\right)$ as in the statement of Theorem 4.1. If $H$ is a divisor such that the linear system $\mathcal{L}_{H}(\mathcal{K})$ is not empty then:

$$
\left(D_{\mathcal{F}}+H-C\right) \cdot C_{1} \geq 0,
$$

where $D_{\mathcal{F}}$ is a Cartier divisor in the divisor class defined by the transition functions $g_{i j}$ associated with the foliation (see the beginning of the section) and $C_{1}$ is the reduced curve consisting of the components of $C$ not contained in the support of $H$. 
Remark 4.1. If we take, in Theorem 4.2, $X=\mathbb{P}^{2}$, then one has that $D_{\mathcal{F}}=(\operatorname{deg}(\mathcal{F})+2) L$, where $L$ is a line. Setting $d=\operatorname{deg}(H)$ one obtains the inequality $(\operatorname{deg}(\mathcal{F})+2+d-\operatorname{deg}(C)) \operatorname{deg}(C) \geq 0$, that is, $\operatorname{deg}(C) \leq$ $\operatorname{deg}(\mathcal{F})+2+d$. Hence, one recovers Theorem 4.1.

Also, a generalization of Theorem 4.1 for 1-dimensional foliations on the projective space $\mathbb{P}^{n}$ is given in [6], also expressed in terms of infinitely near points. The above results have been improved by Esteves and Kleiman in [23] using arguments which do not involve infinitely near points. A recent result concerning the Poincaré problem is given by $\mathrm{C}$. Galindo and the third author in [29]. In it, it is provided an algorithm to decide whether a foliation of $\mathbb{P}^{2}$ has a rational first integral and to compute it in the affirmative case. This algorithm runs whenever we assume the polyhedrality of the cone of curves of the surface obtained by blowing-up the configuration dicritical points in $\mathcal{C}_{\mathcal{F}}$, which we shall denote by $\mathcal{B}_{\mathcal{F}}$ (this happens, for instance, when this configuration is $\mathrm{P}$-sufficient). The inputs of the algorithm are the projective differential 1-form $\Omega$ defining the foliation, the configuration $\mathcal{B}_{\mathcal{F}}$ of dicritical points and the non-dicritical exceptional divisors coming from $\mathcal{B}_{\mathcal{F}}$. We shall explain now the main ideas that give rise to that result. Assume now that $\mathcal{F}$ is a foliation of $\mathbb{P}^{2}$ and let $\pi_{\mathcal{F}}: Z_{\mathcal{F}} \rightarrow \mathbb{P}^{2}$ be the composition of blowing-ups of the configuration $\mathcal{B}_{\mathcal{F}}$. We shall also assume that the cardinality of $\mathcal{B}_{\mathcal{F}}$ is greater than 1 . If $\mathcal{F}$ has a rational first integral one has the following fundamental facts:

(1) A rational first integral $R$ can be taken to be the quotient of two homogeneous polynomial of the same degree $d, F$ and $G$, such that the pencil $\varrho \subseteq\left|\mathcal{O}_{\mathbb{P}^{2}}(d)\right|$ that they provide is irreducible.

(2) $\mathcal{B}_{\mathcal{F}}$ coincides with the configuration of base points $\mathcal{C}_{\mathcal{I}(\varrho)}$ of the pencil $\varrho$ [29, Prop. 1].

(3) The algebraic solutions of $\mathcal{F}$ are the integral components of the curves in the pencil $\varrho$ and the images of their strict transforms on $A\left(Z_{\mathcal{F}}\right)$, together with the strict transforms of the vertical exceptional divisors, generates the face of $N E\left(Z_{\mathcal{F}}\right)$ given by $G_{\varrho}^{\perp} \cap$ $N E\left(Z_{\mathcal{F}}\right)$ see Section 3.3, after Lemma 3.1). Moreover, the vertical exceptional divisors are exactly the non-dicritical exceptional divisors (as a consequence of [30, Prop. 2.5.2.1] and [14, Exercise $7.2])$.

Set $n$ the cardinality of the configuration $\mathcal{B}_{\mathcal{F}}$ (then, $\operatorname{dim} A\left(Z_{\mathcal{F}}\right)=n+1$ ).

Definition 4.1. An independent system of algebraic solutions for $\mathcal{F}$ will be a set $S=\left\{C_{1}, \ldots, C_{s}\right\}$ such that the system

$$
\mathcal{A}_{S}:=\left\{\tilde{C}_{1}, \ldots, \tilde{C}_{s}, E_{i_{1}}, \ldots, E_{i_{n-s}}\right\} \subseteq A\left(Z_{\mathcal{F}}\right)
$$


is $\mathbb{R}$ - linearly independent, where $\tilde{C}_{i}$ denotes the strict transform of $C_{i}$ on $Z_{\mathcal{F}}$ and $\left\{E_{i_{k}}\right\}_{k=1}^{n-s}$ are the strict transforms of the non-dicritical exceptional divisors corresponding to points in $\mathcal{B}_{\mathcal{F}}$.

If the cone of curves $N E\left(Z_{\mathcal{F}}\right)$ is polyhedral and $\mathcal{F}$ has a rational first integral one has that the face $G_{\varrho}^{\perp} \cap N E\left(Z_{\mathcal{F}}\right)$ has codimension 1 and, therefore, it is spanned by $n \mathbb{R}$-linearly independent generators of extremal rays. Moreover, due to the polyhedrality of $N E\left(Z_{\mathcal{F}}\right)$ and the inclusion $Q\left(Z_{\mathcal{F}}\right) \subseteq N E\left(Z_{\mathcal{F}}\right)$, one has that a ray in $A\left(Z_{\mathcal{F}}\right)$ is an extremal ray of $N E\left(Z_{\mathcal{F}}\right)$ if and only if it is spanned by the image in $A\left(Z_{\mathcal{F}}\right)$ of an integral curve on $Z_{\mathcal{F}}$ with strictly negative self-intersection. Therefore, we can conclude the following

Proposition 4.1. If $N E\left(Z_{\mathcal{F}}\right)$ is polyhedral and $\mathcal{F}$ has a rational first integral then there exists an independent system of algebraic solutions $S$ such that $\tilde{C}^{2}<0$ for all $C \in S$. Moreover, the hyperplane $G_{\varrho}^{\perp}$ is generated by $\mathcal{A}_{S}$.

We assume from now on that $N E\left(Z_{\mathcal{F}}\right)$ is a polyhedral cone. The above mentioned algorithm consists of two parts. In the first one, from the data $\left\{\Omega, \mathcal{B}_{\mathcal{F}},\left(E_{i_{1}}, \ldots, E_{i_{n-s}}\right)\right\}$ (which comes from the resolution of the singularities of $\mathcal{F}$ ), either one concludes that $\mathcal{F}$ has no rational first integral, or an independent system of algebraic solutions is returned [29, Alg. 3]. The algorithm generates a strictly increasing sequence of convex cones $V_{0} \subset V_{1} \subset \cdots$ such that $V_{0}$ is generated by $\left\{E_{Q}\right\}_{Q \in \mathcal{B}_{\mathcal{F}}}$ and $V_{i}$ is generated by $V_{i-1} \cup\left\{\tilde{Q}_{i}\right\}$ for $i \geq 1$, where $Q_{1}, Q_{2}, \ldots$ are curves on $\mathbb{P}^{2}$ (ordered with non-decreasing degrees) satisfying certain conditions, being $\tilde{Q}_{i}^{2}<0$ among them. We stop when one of the following cases occurs: (1) there exists a subset $S$ of $\left\{Q_{1}, \ldots, Q_{i}\right\}$ which is an independent system of algebraic solutions, or (2) $Q\left(Z_{\mathcal{F}}\right) \subseteq V_{i}$. If case (2) holds but (1) does not occur, then we conclude that $\mathcal{F}$ has no rational first integral (see the explanation of Algorithm 3 of [29]). The second part of the algorithm [29, Alg. 2] will be applied when, in the first part, an independent system of algebraic solutions $S$ has been obtained. In the case that $\mathcal{F}$ had rational first integral, the hyperplane $G_{\varrho}^{\perp}$ would be the one generated by $\mathcal{A}_{S}$. Hence, the first step will be to compute the primitive (in the lattice $\mathbb{Z}^{n+1} \cong \operatorname{Pic}\left(Z_{\mathcal{F}}\right) \subseteq A\left(Z_{\mathcal{F}}\right)$ ) class $T_{\mathcal{F}, S}$ such that the hyperplane $T_{\mathcal{F}, S}^{\perp}$ is the one generated by $\mathcal{A}_{S}$ and $T_{\mathcal{F}, S} \cdot \pi_{\mathcal{F}}^{*} L>0$ for a line $L$ (see [29, page 618]). Notice that if $\mathcal{F}$ had a rational first integral then $G_{\varrho}$ should be equal to $\alpha T_{\mathcal{F}, S}$ for some integer $\alpha>0$. By [29, Prop. 4] one of the following conditions is satisfied:

(1) $T_{\mathcal{F}, S}^{2} \neq 0$. In this case $\mathcal{F}$ has not a rational first integral (since, otherwise, the equality $G_{\varrho}^{2}=0$ gives a contradiction). 
(2) The coefficients of all the elements of $\mathcal{A}_{S}$ in the decomposition of $T_{\mathcal{F}, S}$ as linear combination of $\mathcal{A}_{S}$ are strictly positive. In this case $[29$, Th. 2] shows the existence of a unique possible value for $\alpha$. If the dimension of the space $H^{0}\left(\mathbb{P}^{2}, \pi_{\mathcal{F}_{*}} \mathcal{O}_{Z_{\mathcal{F}}}\left(\alpha T_{\mathcal{F}, S}\right)\right)$ is not 2 , then $\mathcal{F}$ has not a rational first integral (otherwise we have a contradiction, since $V_{\varrho}$ would coincide with this space by Prop. 3.4). If the above dimension is 2 , then one can compute a basis $\{F, G\}$ of the space. If $\Omega \wedge d(F / G)=0$ then $F / G$ is a first integral; otherwise, $\mathcal{F}$ is not algebraically integrable.

(3) The set $\left\{\lambda \in \mathbb{Z}_{+} \mid h^{0}\left(\mathbb{P}^{2}, \pi_{\mathcal{F}_{*}} \mathcal{O}_{Z_{\mathcal{F}}}\left(\lambda T_{\mathcal{F}, S}\right)\right) \geq 2\right\}$ is not empty. In this case one can take $\alpha$ to be the minimum of this set and proceed as in the above case.

Remark 4.2. Although the algorithm is expressed, for clarity, in terms of divisors, it involves the computation of linear systems of plane curves coming from clusters. For instance, to find the curves $Q_{1}, Q_{2}, \ldots$ one takes clusters $\mathcal{K}=\left(\mathcal{B}_{\mathcal{F}}, \underline{m}\right)$ and, beginning with $d=1$ and increasing $d$ successively, computes (for each fixed value of $d$ ) all the linear systems $\mathcal{L}_{d}(\mathcal{K})$ with $(d ; \underline{m})$ satisfying certain properties: $d^{2}-\sum m_{i}^{2}<0$, the proximity inequalities and other properties coming from the adjunction formula. These properties come from the fact that we want that $\tilde{Q}_{i}$ be linearly equivalent to $d \pi \mathcal{F}^{*} L-D(\mathcal{K})$ ( $L$ being a general line). The computation of basis of the linear systems involves the resolution of systems of linear equations. We are finding non-empty linear systems $\mathcal{L}_{d}(\mathcal{K})$ (whose projective dimension will be, a fortiori, equal to 0 ) whose unique curve $Q_{i}$ passes effectively through the cluster $\mathcal{K}$.

4.2. Applications to the Harbourne-Hirschowitz Conjecture. Clusters of infinitely near points have been applied also to obtain results dealing with the so-called Harbourne-Hirschowitz Conjecture and related problems. Fixing $r+1$ points $P_{0}, P_{1}, \ldots, P_{r}$ of $\mathbb{P}^{2}$ in general position and given $r+1$ non-negative integers $\underline{m}=\left(m_{0}, m_{1}, \ldots, m_{r}\right)$, the linear system $\mathcal{L}_{d}(\underline{m})$ of plane projective curves of fixed degree $d$ having multiplicity $m_{i}$ (or larger) at $P_{i}$ for each $i$, has an expected dimension (attained when all the conditions being imposed are independent):

$$
\operatorname{edim} \mathcal{L}_{d}(\underline{m}):=\max \left\{\frac{d(d+3)}{2}-\sum_{i=0}^{n} \frac{m_{i}\left(m_{i}+1\right)}{2},-1\right\} .
$$

Those systems whose dimension is larger than the expected one are called special. The Harbourne-Hirschowitz Conjecture intends to give a description of all special linear systems. One of the equivalent formulations of this conjecture asserts that a linear system is special if and only if it has a multiple fixed component such that its strict transform on the surface 
obtained by blowing-up the points $P_{0}, P_{1} \ldots, P_{r}$ is a (-1)-curve. This conjecture goes back to B. Segre [65] and it has been reformulated by several authors (see [36], [31], [40], [38], [17], [18], and [16] for a survey). There exists an extensive literature either giving partial proofs of the conjecture or dealing with related subjects. It is out of the scope of this paper to give a global overview of the topic; [37], [56], [16] and references given therein will be helpful for the interested reader. We mention here the result given in [61], where the semicontinuity theorem and a sequence of specializations to constellations of infinitely near points are used to obtain an algorithm for computing an upper bound for the least degree $d$ for which $r+1 \geq 9$ general points of given multiplicities $m_{0}, m_{1}, \ldots, m_{r}$ impose independent conditions to the linear system of curves of degree $d$ (that is, the regularity of the system of multiplicities); also, an explicit formula for a bound is obtained when all the multiplicities are equal to $m: d+2 \geq(m+1)(\sqrt{r+2.9}+\pi / 8)$ (the Harbourne-Hirschowitz Conjecture implies that the imposed conditions are independent when $d(d+3) \geq(r+1) m(m+1)-2)$. In [54] it is provided an unbounded family of systems of multiplicities $\left(m_{i}\right)_{i=0}^{r}$ for which the Harbourne-Hirschowitz Conjecture is satisfied (considering $\mathbb{C}$ as the base field). This result is obtained specializing the $r+1$ general points to the configuration of base points of the pencil at infinity defined by an Abhyankar-Moh-Suzuki curve and using semicontinuity. The statement is the following one:

Theorem 4.3. Let $\varrho$ be the pencil at infinity defined by an AbhyankarMoh-Suzuki curve that is not a line and let $\mathcal{C}_{\mathcal{I}(\varrho)}=\left\{Q_{0}, Q_{1}, \ldots, Q_{r}\right\}$ be its constellation of base points. Let $\underline{m}=\left(m_{0}, m_{1}, \ldots, m_{r}\right)$ be a system of multiplicities such that the cluster $\left(\overline{\mathcal{C}_{\mathcal{I}}(\varrho)}, \underline{m}\right)$ satisfies the proximity inequalities $m_{i}-\sum_{Q_{j} \rightarrow Q_{i}} m_{j} \geq 0,0 \leq i \leq r$, the second one being a strict inequality (that is, $m_{1}-\sum_{Q_{j} \rightarrow Q_{1}} m_{j}>0$ ). If the linear system (supported at general points) $\mathcal{L}_{d}(\underline{m})$ is special, then it has a multiple fixed component whose strict transform on the surface obtained by blowing-up the general points is a (-1)-curve. Furthermore, this curve is the line joining the points corresponding with the multiplicities $m_{0}$ and $m_{1}$.

Notice that the above result depends only on the P-Enriques diagram associated with the resolution of the singularity at infinity of the fixed Abhyankar-Moh-Suzuki curve, and not on the curve itself. These P-Enriques diagrams are completely characterized (see [24] and [25]) and each of them provides an unbounded family of multiplicities for which the Harbourne-Hirschowitz Conjecture is satisfied. It is worth adding that some of the facts in which the proof of Theorem 4.3 is based are the above mentioned results (Theorems 3.2 and 3.3) on the structure of the effective semigroup, the nef cone and the characteristic cone of the surface obtained 
by eliminating the base points of a pencil defined by a curve having one place at infinity. In addition, it is relevant the fact that the configurations $\mathcal{C}_{\mathcal{I}(\varrho)}$ as in the statement of Theorem 4.3 are P-sufficient (Prop. 3.6). In [54] it is also generalized the algorithm given in [61] for bounding the regularity of a system of multiplicities by using P-Enriques diagrams of pencils at infinity associated with Abhyankar-Moh-Suzuki curves.

\section{References}

[1] S. S. Abhyankar, Lectures on expansion techniques in Algebraic Geometry, Tata Institute of Fundamental Research Lectures on Mathematics and Physics 57, Tata Institute of Fundamental Research, Bombay (1977).

[2] S. S. Abhyankar, T. T. Moh, Newton-Puiseux expansion and generalized Tschirnhausen transformation, J. Reine Angew. Math. 260 (1973), 47-83 and 261 (1973), $29-54$.

[3] S. S. Abhyankar, T. T. Moh, Embeddings of the line in the plane, J. Reine Angew. Math. 276 (1975), 148-166.

[4] A. Beauville, Complex algebraic surfaces, London Mathematical Society Student Texts 34 (1996).

[5] A. Campillo, M. Carnicer, Proximity inequalities and bounds for the degree of invariant curves by foliations of $\mathbb{P}_{\mathbb{C}}^{2}$, Trans. Amer. Math. Soc. 349 (9) (1997), $2211-2228$

[6] A. Campillo, M. Carnicer, J. García de la Fuente, Invariant curves by vector fields on algebraic varieties, J. Lond. Math. Soc. II. Ser. 62 (1) (2000), 56-70.

[7] A. Campillo, C. Galindo, J. García de la Fuente, A. Reguera, On proximity and intersection inequalities for foliations on algebraic surfaces, Journés singulières et Jacobiennes, Institut Fourier (1993), 25-40.

[8] A. Campillo, G. Gonzalez-Sprinberg, On Characteristic Cones, Clusters and Chains of Infinitely Near Points, Progress in Math. Vol. 162, Birkhäuser (1998), 251-261.

[9] A. Campillo, G. Gonzalez-Sprinberg, M. Lejeune-Jalabert, Clusters of infinitely near points, Math. Ann. 306 (1996), 169-194.

[10] A. Campillo, O. Piltant, A. J. Reguera, Cones of curves and of line bundles on surfaces associated with curves having one place at infinity, Proc. London Math. Soc. 84 (2002), 559-580.

[11] A. Campillo, O. Piltant, A. Reguera, Cones of curves and of line bundles "at infinity", J. Algebra 293 (2005), 513-542.

[12] M. Carnicer, The Poincaré problem in the nondicritical case, Ann. Math. 140 (1994), 289-294.

[13] E. Casas, Infinitely near imposed singularities, Math. Ann. 287 (1990), 429-454

[14] E. Casas, Singularities of plane curves, London Math. Soc. Lecture Note Series 276 (2000), Cambridge University Press.

[15] D. Cerveau, A. Lins-Neto, Holomorphic foliations in $\mathbb{C P}(2)$ having an invariant algebraic curve, Ann. Inst. Fourier 41 (4) (1991), 883-903.

[16] C. Ciliberto, Geometric aspects of polynomial interpolation in more variables and of Waring's problem, Progr. Math 201 (2001), Birkhäuser, Basel.

[17] C. Ciliberto, R. Miranda, Degenerations of planar linear systems, J. Reine Angew. Math. 501 (1998), 191-220.

[18] C. Ciliberto, R. Miranda, Linear systems of plane curves with base points of equal multiplicity, Trans. Amer. Math. Soc. 352 (2000), no. 9, 4037-4050. 
[19] C. D'Cruz, Integral closedness of MI and the formula of Hoskin and Deligne for finitely supported complete ideals, J. Algebra 304 (2006), 613-632.

[20] S. D. Cutkosky, Complete Ideals in Algebra and Geometry, Contemporary Math. Vol.159 (1994), 27-39.

[21] F. Delgado de la Mata, A factorization theorem for the polar of a curve with two branches, Compositio Math. 92 (1994), 327-375.

[22] F. Enriques, O. Chisini, Lezioni sulla teoria geometrica delle equazioni e delle funzioni algebriche, Libro IV, (1915) (N. Zanichelli reprint, Bologna 1985).

[23] E. Esteves, S. Kleiman, Bounds on leaves of one-dimensional foliations, Bull. Braz. Math. Soc. (N.S.) 34 (1) (2003), 145-169.

[24] J. Fernández de Bobadilla, A new geometric proof of Jung's theorem on factorisation of automorphisms of $\mathbb{C}^{2}$, Proc. Amer. Math. Soc. 133 (2005), 15-19.

[25] J. Fernández de Bobadilla, Moduli spaces of polynomials in two variables, Mem. Amer. Math. Soc. 173 (2005), no. 817.

[26] J. W. Gaddum, Linear inequalities and quadratic forms, Pacific J. Math. 8 (1958), 411-414.

[27] C. Galindo, F. Monserrat, The cone of curves associated to a plane configuration, Comment. Math. Helv. 80 (2005), 75-93.

[28] C. Galindo, F. Monserrat, On the cone of curves and of line bundles of a rational surface, Internat. J. Math. 15 (4) (2004), 393-407.

[29] C. Galindo, F. Monserrat, Algebraic integrability of foliations of the plane, J. Diff. Equations 231 (2006), no. 2, 611-632.

[30] J. Garcia de la Fuente, Geometría de los sistemas lineales de series de potencias en dos variables, $\mathrm{Ph}$. D. thesis, Valladolid University (1989) (in Spanish).

[31] A. Gimigliano, On linear systems of plane curves, Thesis, Queen's University, Kingston (1987).

[32] A. Gimigliano, Our thin knowledge of fat points, Queen's Papers in Pure and Applied Math. 83, The Curve Seminar at Queen's, Vol IV, Queen's University, kingston, Canada (1989).

[33] X. Gómez-Mont, L. Ortiz, Sistemas dinámicos holomorfos en superficies, Aportaciones Matemáticas 3, Sociedad Matemática Mexicana, 1989 (in Spanish).

[34] G. Gonzalez-Sprinberg, A. Pereyra, Sobre diagramas de Enriques y constelaciones tóricas, Publ. Mat. Uruguay, 8, 1999, 113-138 (in Spanish).

[35] G. Gonzalez-Sprinberg, Generalized Enriques Diagrams and Characteristic Cones, Advanced Studies in Pure Math, 29, 2000, 115-134.

[36] B. Harbourne, The geometry of rational surfaces and Hilbert functions of points in the plane, Can. Math. Soc. Conf. Proc. 6 (1986), 95-111.

[37] B. Harbourne, Problems and progress: a survey on fat points in $\mathbb{P}^{2}$, Zerodimensional schemes and applications (Naples, 2000), Queen's Papers in Pure and Appl. Math. 123 (2002), 85-132.

[38] B. Harbourne, Points in good position in $\mathbb{P}^{2}$, Zero-dimensional schemes (Ravello, 1992), 213-229, de Gruyter, Berlin (1994).

[39] R. Hartshorne, Algebraic geometry, GTM 52, Springer-Verlag, 1987.

[40] A. Hirschowitz, Une conjecture pour la cohomologie des diviseurs sur les surfaces rationnelles génériques, J. Reine Angew. Math. 397 (1989), 208-213.

[41] E. Hyry, Y. Nakamura, L. Ojala, Adjoint ideals and Gorenstein blowups in two dimensional regular local rings, Math. Z. 254 (4) (2006), 767-783.

[42] G. Kempf, F. Knudsen, D. Mumford, B. Saint Donat, Toroidal embeddings I, LNM 339, Springer-Verlag (1993)

[43] S. Kleiman, Toward a numerical theory of ampleness, Annals of Math. 84 (1966), 293-344.

São Paulo J.Math.Sci. 3, 1 (2009), 115-160 
[44] J. Kollár, Rational curves on algebraic varieties, Ergeb. Math. Grenzgeb. (3) 32, Springer-Verlag, 1996.

[45] A. Lemahieu, W. Veys, On monodromy for a class of surfaces, C. R. Acad. Sci. 345/11 (2007), 633-638.

[46] A. Lemahieu, W. Veys, Zeta functions and monodromy for surfaces that are general for a toric idealistic cluster, Int. Math. Res. Notices (2009), No. 1, 11-62

[47] A. Lins-Neto, Some examples for the Poincaré and Painlevé problems, Ann. Sc. Éc. Norm. Sup. 35 (2002), 231-266.

[48] J. Lipman, Rational singularities with applications to algebraic surfaces and unique factorization, Publ. IHES 36 (1969), 195-279.

[49] J. Lipman, On complete ideals in regular local rings, In: Algebraic Geometry and Commutative Algebra in Honor of M. Nagata, Kinokuniya (1987), 203-231.

[50] J. Lipman, Adjoints and polars of simple complete ideals in two dimensional regular local rings, Bull. Soc. Math. de Belgique 45 (1993), 223-244.

[51] J. Lipman, Proximity inequalities for complete ideals in two-dimensional regular local rings, Contemporary Math. 159 (1994), 293-306.

[52] J. Lipman, A vanishing theorem for finitely supported ideals in regular local rings, Michigan Math. J. 57 (2008), 573-585.

[53] Y. Manin, Cubic forms. Algebra, Geometry, Arighmetic. North Holland Mathematical Library 4, North Holland, Amsterdam, London (1974).

[54] F. Monserrat, Curves having one place at infinity and linear systems on rational surfaces, J. Pure Appl. Algebra, 211 (2007), 685-701.

[55] D. McDuff, L. Polterovich, Symplectic packings and algebraic geometry, Inventiones Math. 115 (1994), 405-429.

[56] R. Miranda, Linear systems of plane curves, Notices of the Amer. Math. Soc. 46 (2) (1999), 192-202.

[57] T. T. Moh, On analytic irreducibility at $\infty$ of a pencil of curves, Proc. Amer. Math. Soc. 44 (1974), 22-23.

[58] M. Nagata, On the 14-th problem of Hilbert, Amer. J. Math. 33 (1959), 766-772.

[59] T. Oda, Convex bodies and algebraic geometry, an introduction to the theory of toric varieties, Ergebnisse der Math. 15, Springer-Verlag (1988).

[60] H. Poincaré, Sur l'intégration algébrique des équations différentielles du premier ordre et du premier degré, Rend. Circ. Mat. Palermo 5 (1891), 161-191.

[61] J. Roé, Linear systems of plane curves with imposed multiple points, Illinois J. Math. 45 (2001), no. 3, 895-906.

[62] J. Roé, Conditions imposed by tacnodes and cusps, Trans. Amer. Math. Soc. 353 (2001), no. 12, 4925-4928.

[63] J. Roé, Varieties of clusters and Enriques diagrams, Math. Proc. Cambridge Phil. Soc. 137 (2004), no. 1, 69-94.

[64] A. Sathaye, On planar curves, Amer. J. Math. 99 (1977), no. 5, 1105-1135.

[65] B. Segre, Alcune questioni su insiemi finiti di punti in geometria algebrica, Atti Convegno Intern. di Geom. Alg. di Torino (1961), 15-33.

[66] A. Seidenberg, Reduction of singularities of the differentiable equation Ady = Bdx, Amer. J. Math. 90 (1968), 248-269.

[67] M. Suzuki, Affine plane curves with one place at infinity, Ann. Inst. Fourier 49 (1999), no. 2, 375-404.

[68] E. Tostón, Finitely supported monomial complete ideals, preprint, Prepublicaciones del Departamento de Álgebra, Universidad de Valladolid, no, 67 (1997).

[69] G. Xu, Curves in $\mathbb{P}^{2}$ and symplectic packings, Math. Ann. 299 (1994). 
[70] O. Zariski, Polynomial ideals defined by infinitely near base points, Amer. J. Math. 60 (1938), 151-204.

[71] O. Zariski, P. Samuel, Commutative Algebra II, Appendices 4 and 5, Van Nostrand (1960). 